\title{
Taking Another Look at the van der Waals Equation of State - Almost 150 Years Later
}

\author{
Kontogeorgis, Georgios M.; Privat, Romain; Jaubert, Jean-Noel
}

Published in:

Journal of Chemical and Engineering Data

Link to article, DOI:

10.1021/acs.jced.9b00264

Publication date:

2019

Document Version

Publisher's PDF, also known as Version of record

Link back to DTU Orbit

Citation (APA):

Kontogeorgis, G. M., Privat, R., \& Jaubert, J-N. (2019). Taking Another Look at the van der Waals Equation of State - Almost 150 Years Later. Journal of Chemical and Engineering Data, 64(11), 4619-4637.

https://doi.org/10.1021/acs.jced.9b00264

\section{General rights}

Copyright and moral rights for the publications made accessible in the public portal are retained by the authors and/or other copyright owners and it is a condition of accessing publications that users recognise and abide by the legal requirements associated with these rights.

- Users may download and print one copy of any publication from the public portal for the purpose of private study or research.

- You may not further distribute the material or use it for any profit-making activity or commercial gain

- You may freely distribute the URL identifying the publication in the public portal 


\section{Taking Another Look at the van der Waals Equation of State- Almost 150 Years Later}

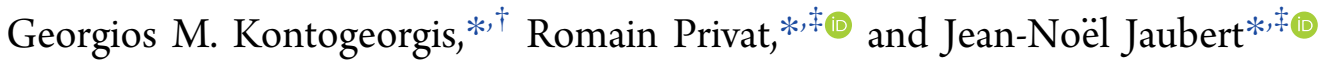

$\dagger$ Center for Energy Resources Engineering (CERE) \& KT-Consortium, Department of Chemical and Biochemical Engineering, Building 229, Technical University of Denmark, Kongens Lyngby, 2800, Denmark

‡Université de Lorraine, École Nationale Supérieure des Industries Chimiques, Laboratoire Réactions et Génie des Procédés (UMR CNRS 7274), 1 rue Grandville, 54000 Nancy, France

\section{Supporting Information}
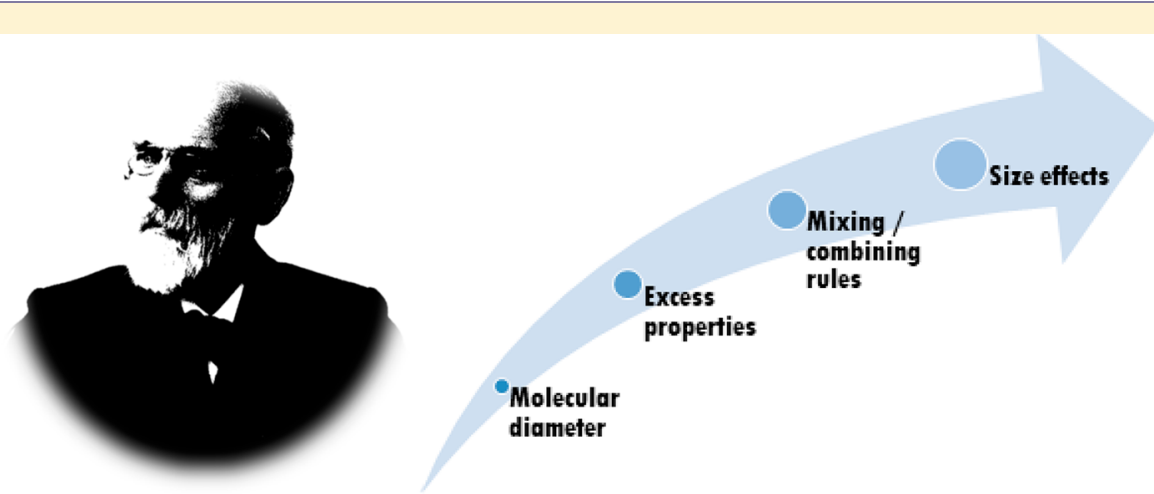

ABSTRACT: The van der Waals (vdW) equation of state has long fascinated researchers and engineers, largely because of its simplicity and engineering flexibility. At the same time as vdW and other equations of state (EoS) have been proposed, at first mostly used for petrochemical applications, solution theories in the form of activity coefficient models have been developed, focusing on the accurate representation of the liquid phase, of interest to the chemical industry. For both the van der Waals (and other cubic EoS) and the activity coefficient models we can identify size and energy terms, although different terminologies have been used (e.g., combinatorial-free volume, entropic, excess entropy or repulsive terms on one side and "residual", energetic, excess energy or attractive contributions on the other side). These definitions are not necessarily identical, as it will be shown here, and the identification of distinct separable contributions in thermodynamic models is not always straightforward. Moreover, the different traditions lead sometimes to confusion as to the actual range of applicability of these models, and it may be useful to consider them (EoS and activity coefficient models) together. While much has been written about the van der Waals equation of state, a particular insight is obtained when the model is expressed in terms of excess Gibbs energy and activity coefficient expressions. We show that such a transformation, analysis of the distinct size and energy terms of $\mathrm{vdW}$ and comparison to classical solution theories, provides insight into the physical meaning, capabilities, and limitations of the model, including the associated mixing and combining rules used. We will discuss how this analysis of the vdW and subsequent equations of state, such as the Soave-Redlich-Kwong and Peng-Robinson, have enhanced our understanding of the actual applicability range of the models in terms of size effects or excess properties. The analysis reveals that the capabilities of the vdW and in general of the vdW-type cubic equations of state are possibly more significant than traditionally considered, and that the task of more advanced models in trying to "beat them (the cubic EoS)", may be more difficult than previously anticipated.

\section{SOME INTRODUCTORY REMARKS}

The van der Waals equation of state (vdW EoS) was presented by van der Waals in his 1873 Ph.D. thesis ${ }^{1}$ and for this he was awarded the Nobel Prize in Physics 1910. The 100 years Prize anniversary (2010) was celebrated among others with a special issue in J. Supercrit. Fluids, also including a history review by Valderrama, ${ }^{2}$ who points out that the well-known form of vdW EoS is not included in his thesis. It was, however, presented later, as well as it is present in his Nobel prize speech. ${ }^{3}$

van der Waals, at the beginning of his Nobel prize speech, ${ }^{3}$ states that
"Thus, I conceived the ideas that there is no essential difference between the gaseous and the liquid state of matter... And so the idea of continuity occurred to me".

His thesis was indeed entitled "On the continuity of the gaseous and liquid state" and this together with Andrew's discovery of the existence of the $\mathrm{CO}_{2}$ critical point ${ }^{4}$ has made

Special Issue: Celebrating Our High Impact Authors

Received: March 25, 2019

Accepted: July 5, 2019

Published: August 2, 2019 
many to connect the two and consider that van der Waals' original target was indeed an understanding of this continuity. Klein $^{5}$ in his excellent account of the historical origins of the vdW equation, is of another opinion. In his view, van der Waals was more concerned (having studied the Laplace work on capillarity and the Clausius work on the second law) to add "molecular concepts" and to continue Newton's uncompleted work on the search for the short-range molecular (nongravitational) forces and their role in determining structure and properties of matter.

The molecular aspects of the van der Waals equation are also evident in his Nobel prize speech, ${ }^{3}$ as close to the end of his speech, he states:

"It will be perfectly clear that in all my studies, I was quite convinced of the real existence of molecules, that I never regarded them as a figment of my imagination... When I began my studies I had the feeling that I was almost alone in holding that view... Many of those who opposed it most have ultimately been won over, and my theory may have been a contributory factor".

The qualitative success-agreement of the theory with many experimental data-was evident quite soon and as Lebowitz and Waisman ${ }^{6}$ wrote in 1980:

"Indeed, George Uhlenbeck has complained that the successes of the van der Waals equation were so remarkable that they practically killed the subject for more than 50 years".

Indeed, the vdW EoS is a successful model, being a representation of the two-parameter corresponding states principle (when the parameters are estimated using critical point data) and it is shown that it can represent five out of the six types of general phase diagrams according to the van Konynenburg and Scott classification. ${ }^{7,8}$

Various researchers ${ }^{9-11}$ have presented rigorous derivations of the vdW EoS based on statistical thermodynamics, where it can also be seen that both parameters of the model have a clear physical significance and can, in principle, be estimated from intermolecular potential constants and molecular diameters. Even though this is rarely done, it does not deprive the model from its fundamental nature, something of which van der Waals himself was aware and quite proud: ${ }^{3}$

"The two constants that appear in the theory have a real physical meaning, namely that of molecular volumes and attraction and no one will deny that the theory will influence progress in this field".

van der Waals was also aware and rather forthcoming of several of the limitations of his theory or issues that demanded clarification. The temperature-dependency of the parameters will be discussed in depth in the next section.

He was aware that, despite the importance of what since has been known as "van der Waals forces", strong interactions could not be accounted for by the vdW EoS. In his Nobel-prize speech, ${ }^{3}$ he stated at various places:

"In fact, bluntly speaking, the result would be an equation of state compatible with experimental data is totally impossible. No such equation is possible, unless something is added, namely that the molecules associate to form larger complexes"

"I have termed it pseudo association to differentiate it from the association which is of chemical origin"

"The number of formed complexes increases with decreasing temperature. At the critical point, so I was compelled to conclude, only a very small part of the weight is present as complexes".

"What is the origin of this complex formation, this pseudo association?"
He does not actually conclude that the origin of complex formation (pseudo association) is hydrogen bonding or Lewis acid-base interactions in more general terms, but we would like to believe that this is what he actually meant!

This was a period where Frederick Dolezalek had insisted ${ }^{12}$ that all nonidealities are due to association-complex formation, not just in the obvious cases of acetic acid dimers or acetone-chloroform strong complexes, but for all mixtures. $\mathrm{He}$ insisted in neglecting the physical interactions, believing that the "chemical theory" can explain all nonidealities, and this resulted in deep polemic between him and the Amsterdam school, esp. van der Waals' student van Laar who wrote among others: ${ }^{13,14}$

"Unfortunately, I have to rob Mr. Dolezalek of many illusions" and then lists ten such illusions, the last being his (van Laar's) illusion that Mr. Dolezalek will be convinced of the indefensibleness of his theory"

Today, most researchers agree that both physical and chemical forces are needed for an appropriate and general representation of solutions; with the chemical forces dominating in hydrogen bonding and related systems, while being essentially zero in nonpolar/polar solutions.

Indeed, in a short manuscript in Proceedings of the National Academy of Sciences, Heidemann and Prausnitz ${ }^{15}$ presented "A van der Waals-type equation of state for fluids with associating molecules" (title of the article), illustrating how a chemicaltype approach can be incorporated into a vdW-type EoS enabling it to model associating fluids. This article in many respects opened up the field of especially the chemical-type approaches developed by Anderko and others in the subsequent years ${ }^{16,17}$ and, about $10-15$ years later, of the advent of the SAFT approach, which was subsequently much used for associating fluids.

Another aspect of the vdW EoS about which van der Waals himself was even more concerned (compared to the lack of accounting for "pseudo-association") was its repulsive term (concern mentioned several times in his Nobel-prize speech). $\mathrm{He}$ recognized that this term was not an optimum representation of the repulsive effects. As Figure 1 shows,

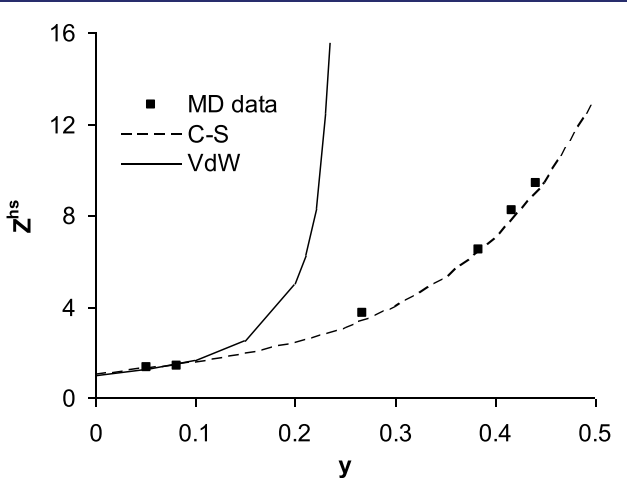

Figure 1. Repulsive part of vdW EoS compared to the CarnahanStarling (C-S) equation ${ }^{18}$ and Molecular Dynamics data. $y$ is the reduced density $b \rho / 4$.

indeed the vdW repulsive term is not in agreement with the simulation data or the equation of state (Carnahan-Starling ${ }^{18}$ ) that represents a hard-sphere fluid. While we are not aware if van der Waals suspected this (probably not!) and even though the hard-sphere fluid only represents part of the repulsions, van der Waals in his Nobel prize speech" ${ }^{3}$ mentioned that "I still 
wonder whether there is a better way, in fact this question continually obsesses me, I can never free myself from it, it is with me even in my dreams".

It is rather interesting that, despite this, it is actually the vdW repulsive term that has remained in the two most widely used cubic EoS today (SRK; Soave; ${ }^{19}$ Peng-Robinson ${ }^{20}$ ), while the attractive term has been modified in more than one way. Mathias and Klotz ${ }^{21}$ have underlined this by stating " $R T /(v-$ b) is wrong, but somehow will exist in the most popular (reliable) models".

One aspect is the cancellation of errors between attractive and repulsive terms (adding the $\mathrm{C}-\mathrm{S}$ term and maintaining the $\mathrm{vdW}$ attractive has not helped ${ }^{22}$ ), but another aspect is the fact that the vdW repulsive term results greatly depend on the covolume parameter used, and as shown in Figure 2; an effective covolume equal to half the "original" covolume brings the vdW term somewhat closer to the $\mathrm{C}-\mathrm{S}$ results.

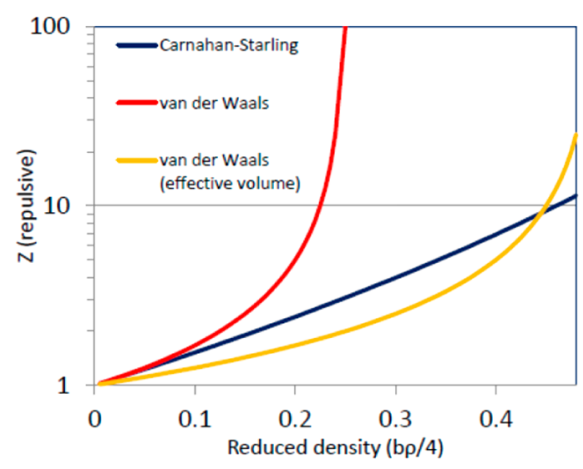

Figure 2. Repulsive part of vdW EoS, with the original covolume and an "effective co-volume" (half of the original covolume) compared to the Carnahan-Starling equation. Figure reprinted with permission from ref 23. Copyright 2016 Technical University of Denmark.

This has been noticed by Wong and Prausnitz ${ }^{24}$ and Prausnitz, ${ }^{25}$ but it was known to van der Waals as well, as he stated in his Nobel-prize speech: ${ }^{3}$

"To my surprise I realized that the amount by which the volume must be reduced is variable, that in the extremely dilute state this amount which I have notated $b$, is 4-fold the molecular volume but that this amount decreases with decreasing external volume and gradually falls to about half. But the law governing this decrease has still not been found!"

In other words, while for dilute solutions and in its original formulation the vdW covolume is given as

$$
b^{\mathrm{vdW}}=\frac{2}{3} \pi N_{\mathrm{A}} \sigma^{3}=4 V_{\text {molecule }}
$$

where $N_{\mathrm{A}}$ is the Avogadro number and $\sigma$ is the molecular diameter. The molecular volume, noted $V_{\text {molecule }}$ in eq 1 is today called the van der Waals volume and will be noted $V_{\mathrm{W}}$ hereafter.

In the effective form, the value is half:

$$
b^{\text {effective }}=\frac{b^{\mathrm{vdW}}}{2}=\frac{1}{3} \pi \mathrm{N}_{\mathrm{A}} \sigma^{3}=2 V_{\text {molecule }}
$$

As mentioned above, van der Waals was well aware of the limitations of the repulsive term of his EoS and also the need for lower values of the covolume compared to those obtained from the theoretical eq 1. For $\mathrm{CO}_{2}$, he recommends in his Nobel prize speech, ${ }^{3}$ a value equal to 0.001565 instead of the "theoretical" value of 0.0023 , that is, a factor of 1.5 instead of 2 in the denominator of eq 2 (note that these values are actually volume ratios in $\mathrm{cm}^{3} / \mathrm{cm}^{3} N T P$ - where NTP is the abbreviation of normal temperature and pressure-and are thus dimensionless).

Based on more rigorous calculations today and experimental values for the $\mathrm{CO}_{2}$ diameter, using eq 1 we could calculate a covolume equal to $77.3 \mathrm{~cm}^{3} / \mathrm{mol}\left(b / V_{\mathrm{w}}=3.8 ; V_{\mathrm{w}}\right.$ is the van der Waals volume), while an effective value according to eq 2 would yield $38.6 \mathrm{~cm}^{3} / \mathrm{mol}\left(b / V_{\mathrm{w}}=1.9\right)$ and a covolume estimated from the critical data (temperature and pressure), the one typically used, see discussion next, has a value equal to $43.4 \mathrm{~cm}^{3} / \mathrm{mol}\left(b / V_{\mathrm{w}}=2.2\right)$.

In practice, neither equation 1 or 2 is used but instead the covolume is estimated from the critical properties. There are two choices for the covolume and three for the energy parameters, as shown below for vdW:

$$
\begin{aligned}
& a\left(V_{c}, T_{c}\right)=\frac{9}{8}\left(R T_{c} V_{c}\right) \quad a\left(P_{c}, T_{c}\right)=\frac{27}{64} \frac{R^{2} T_{c}^{2}}{P_{c}} \\
& a\left(P_{c}, V_{c}\right)=3 P_{c} V_{c}^{2} \quad b\left(V_{c}\right)=\frac{1}{3} V_{c} \\
& b\left(P_{c}, T_{c}\right)=\frac{1}{8}\left(\frac{R T_{c}}{P_{c}}\right)
\end{aligned}
$$

That there are various possibilities to estimate the pure EoS parameters was known from the early days when cubic EoS were used in engineering (see, e.g., Martin ${ }^{26}$ ), but as Vera and $\mathrm{Vera}^{27}$ state "van der Waals made his master move" by adopting the equations which give the parameters as a function of $T_{c}$ and $P_{\mathrm{c}}$ rather than of $V_{\mathrm{c}}$. This was then justified by the fact that critical volume data were more difficult to obtain, but it is interesting to note that even today this is the preferred approach for most widely used cubic EoS such as SRK ${ }^{19,28}$ and PR. ${ }^{20,28}$

van der Waals was aware that the critical volume estimation using the covolume, shown in eq 3 , was not accurate. It is wellknown that this value yields a constant $Z_{\mathrm{c}}$ for the vdW EoS of 0.375 , much higher than most experimental data (0.26-0.28) and even of other cubic EoS (SRK, 0.333; and PR, 0.307). In his Nobel Prize speech van der Waals says: ${ }^{3}$

"... $V_{c}=3 b$. And it can again be seen from this equation that what I have termed the weak point of my theory is actually responsible for the theoretical impossibility of calculating accurately the critical volume. Using an approximation formula for $b I$ was able to determine $V_{c}$ as about $2.2 \mathrm{~b}$ ".

It is interesting that if the vdW EoS could result to such a $V_{c}-b$ relationship (as van der Waals considered to be correct), then the critical compressibility factor would be 0.275 . This is, of course, a "hypothetical" calculation as all equations in eq 3 are connected and directly derived from the application of the vdW EoS to the critical point.

Another interesting observation is that using eq 2 and van der Waals statement about $V_{c}$ and covolume, we can arrive to a relationship between $V_{c}$ and the effective covolume $\left(V_{c}=\right.$ $4.4 b^{\text {eff }}$ ) which is not that far from the relationship between $V_{c}$ and van der Waals volume $\left(V_{\mathrm{w}}\right)$ first presented by Bondi in his book $^{29}\left(V_{\mathrm{c}}=5.3 V_{\mathrm{w}}\right)$ and later rediscovered and further validated by Elbro et al. ${ }^{30}$ The similarity between the two values becomes even more pronounced if we consider Wilson's ${ }^{31}$ statement that the fluid hard-core volume is expected to be around 1.15 times the closed packed/van der 
Waals volume. If we assume that $b^{\text {eff }}$ and fluid hard-core volume are equivalent terms, then van der Waals' hypothesis $\left(V_{\mathrm{c}}=4.4 b^{\text {eff }}\right)$ will lead to a $V_{\mathrm{c}}=5.1 \mathrm{~V}_{\mathrm{w}}$.

When eqs 1 and 3 are combined, some reasonable values are obtained for the molecular dimensions, as shown for some compounds in Table 1.

Table 1. Molecular Diameters from the vdW EoS Estimated Using Critical Properties and the Equation:

$b=\frac{2}{3} \pi N_{\mathrm{A}} \sigma^{3}=0.125 R \frac{T_{\mathrm{c}}}{P_{\mathrm{c}}}$

\begin{tabular}{lcc} 
& \multicolumn{2}{c}{ molecular diameter (nm) } \\
\cline { 2 - 3 } compound & $\begin{array}{c}\text { from vdW EoS using } \\
\text { critical properties }\end{array}$ & $\begin{array}{c}\text { from “Experimental” or quantum- } \\
\text { chemistry calculations }\end{array}$ \\
argon & 0.294 & 0.328 \\
methane & 0.3245 & 0.376 \\
$n$-octane & 0.5733 & 0.7451 \\
$\mathrm{CO}_{2}$ & 0.3252 & 0.3941 \\
methanol & 0.331 & 0.3626
\end{tabular}

It is difficult to identify what is the "correct" value of the covolume parameter to be used with vdW (or any other cubic EoS for that matter) as this will depend on the application of interest. The choice of the covolume will, among others, determine the accuracy of the representation of the critical point (eq 3 ) and is expected to affect the density calculations from the model.

For example, let us consider six representative molecules (propane, pentane, decane, hexadecane, benzene, and argon). If the covolume is estimated using eq 1 (with diameters from experimental values), then the ratio between covolume and van der Waals volume $V_{\mathrm{w}}$ (as calculated from Bondi's approach ${ }^{29}$ ) is, for these molecules, 3.4-4.5. This ratio is much smaller, 23 , if the covolume is estimated from the critical temperature and pressure, eq 3 . We like this smaller ratio more, as the hardcore volume/van der Waals volume ratios which correspond to the face-centered cubic (FCC) structure (co-ordination number 12 ) is 1.35 , and it rises to 1.41 for co-ordination number 10, which is an acceptable value for the liquid phase.

The vdW EoS parameters have not been estimated so far from a simultaneous regression of vapor pressure and liquid density data, an approach which has been used for CPA and SAFT models. We can mention ${ }^{32}$ that for the same six compounds mentioned above the $b / V_{\mathrm{w}}$ ratio for CPA (SRK with fitted covolume and energy) is $1.5-1.7$, even closer to the "theoretical ratios" of 1.41 or 1.35 .

It is interesting to note that, as shown by several researchers $^{33-37}$ and for both SRK and PR, the energy and covolume parameters for alkanes (and other compounds) increase linearly with the molecular weight or van der Waals volume when these parameters are fitted (as in the SAFT approach) from vapor pressure and liquid density data. This is not the case, that is, the linear trends are not observed as clearly, when the parameters are obtained from the critical point. Even for heavier alkanes $\left(\mathrm{C}_{16} \ldots \mathrm{C}_{36}\right)$ the $b / V_{\mathrm{w}}$ ratios obtained with the covolume values of Ting et al. ${ }^{33}$ and Voutsas et al. $^{34}$ are of the order 1.7-1.8, much lower than those obtained with the same models using covolumes estimated from the critical point (over 2, close to 3 for heavier alkanes).

Let us also note that in a paper devoted to the reliability of EoS at high pressures, Polishuk ${ }^{38}$ highlighted that the key to successfully correlate very high-pressure data is in fact an appropriate representation of the ratio between the covolume and the liquid volume at saturation.

In all the above discussion, the covolume parameter has been considered constant, independent of temperature. Indeed, this is the usual practice but as Deiters, ${ }^{39}$ and other researchers have shown, accurate representation of caloric properties and several features such as maxima of the virial coefficients, $C_{p}$ minima (inflection curves) or the Joule inversion curves require "softer repulsions" which can be obtained by temperature $(T)$-dependent covolumes. While this is indeed correct, as Polishuk and co-workers ${ }^{40}$ emphasize, great care should be exercised, as with T-dependent covolume parameters there is danger for obtaining negative heat capacities or negative virial coefficients at high temperatures. Finding an appropriate $T$-dependency for the covolume parameter (both physically correct and engineeringly useful) is not a trivial thing and this may be the reason why this path is not pursued in most successful engineering cubic equations of state.

Finally, we wish to emphasize that it is not our target to provide in this work a comprehensive review of vdW or other cubic EoS; for this we recommend the recent manuscript by Lopez-Echeverry et al. ${ }^{41}$ and the earlier ones by Valderrama, ${ }^{42}$ Wei and Sadus, ${ }^{43}$ and Ghosh ${ }^{44}$ and from a more historical point of view the excellent review of Gubbins. ${ }^{14}$ The legacy of van der Waals and its significance in science and engineering, even up to the period before the advent of the modern cubic EoS (SRK and PR), is presented in an excellent short review in Nature by Rowlinson. ${ }^{45}$ Our purpose in this work is to analyze some characteristics of vdW (and other vdW-type cubic EoS) which can offer an insight into some of the important capabilities and may also point out some limitations. In some sense, our manuscript adds one more angle to look at the model, in addition to the "Thirteen ways of looking at the van der Waals equation” presented by Abbott in $1989 .^{46}$

\section{EXCESS PROPERTIES FROM THE VAN DER WAALS EQUATION OF STATE}

The van der Waals equation of state (vdW EoS) is well-known in its usual form:

$$
P=\frac{R T}{V-b}-\frac{a}{V^{2}}
$$

The two parameters, $a$ and $b$, can be estimated from the critical point and considered constants (specific for each substance) or can be left variable, dependent on temperature. Despite the fact that the former is what is mostly considered or referred to in textbooks, the latter (i.e., letting the energy parameter and sometimes even the covolume parameter) being functions of temperature is clearly a possibility and may even be the correct way to do it, as van der Waals himself admitted in his Nobelprize speech more than 100 years ago: ${ }^{3}$

"I have never been able to consider that the last word had been said about the equation of state and I have continually returned to it during other studies. As early as 1873 I recognized the possibility that $a$ and $b$ might vary with temperature, and it is well-known that Clausius even assumed the value of a to be inversely proportional to the absolute temperature".

Even in this 1910 speech he was surprised that others considered these parameters to be constant. He said: ${ }^{3}$

"I never expected this equation, with $a$ and $b$ assigned a constant value to give results numerically in agreement with experiment and yet people almost always thought that were my opinion. This 
astonishes me as in my treatise of 1873 not only did I expressly emphasize the variability of $b$ but also quoted a series of b-values from Andrew's experiment in which for small volumes the change in $b$ with the volume is calculated".

Sandler ${ }^{11}$ presented two alternative derivations of the van der Waals equation of state and in both cases, even under simplifying assumptions, the energy parameters obtained are functions of temperature (with an exponential dependency).

Letting the energy parameter of $\mathrm{vdW}$ be a function of temperature in order to reproduce vapor pressures, has been indeed adopted in modern approaches with the vdW EoS for example, the vdW-711 by Tassios and co-workers ${ }^{47}$ and the vdW EoS with advanced mixing rules by Soave. ${ }^{48}$ Tassios $^{47}$ showed that the vdW EoS with suitable T-dependency of the energy parameters yields for all alkanes (from methane to $n$ $\mathrm{C}_{20}$ ) excellent vapor pressures, with deviations around $2 \%$, entirely comparable to what is obtained by the PengRobinson equation of state. A similar conclusion was reached by Vidal and Bogdanic ${ }^{49}$ where vdW, SRK, and PR behave similarly for a series of $n$-alkane vapor pressures, if the energy parameter is estimated the same way using a Soave-type temperature dependency. Equally good results are obtained for volumes, as shown by Tassios, using a $T$-dependent volume translation in vdW, actually better than SRK and PR. These modern vdW approaches are also discussed later, in the mixing rules section.

In some of our previous works ${ }^{50,51}$ we have presented the $\mathrm{vdW}$ EoS expressed in the forms of excess Gibbs or Helmholtz energies and even activity coefficients (depending on mixing rules). We take another look at these expressions, considering also that the energy parameter may be $T$-dependent and we will analyze the separability of energy and size contributions in the vdW EoS. The latter distinction is not as clear as previously anticipated, as we will show below, and is more complicated when the energy parameter depends on temperature. The superscripts $\mathrm{E}$ and res stand for excess and residual properties, in the usual rigorous thermodynamic definition (i.e., different from the "residual" contributions to activity coefficients used in solution theories, discussed later).

It is rather straightforward to obtain the $g^{\mathrm{E}}$ from vdW using the general equation:

$$
\frac{g^{\mathrm{E}}}{R T}=\ln \varphi-\sum x_{i} \ln \varphi_{i}=\frac{g^{\mathrm{res}}}{R T}-\sum x_{i} \frac{g_{i}^{\text {res }}}{R T}
$$

The fugacity coefficient expression for vdW, either for a pure compound or the mixture, is given by the equation:

$$
\ln \varphi=-\ln \left(\frac{P(V-b)}{R T}\right)+\left(\frac{P V}{R T}-1\right)-\frac{1}{R T} \frac{a}{V}
$$

Combining eqs 5 and 6, we get after some algebra:

$$
\begin{aligned}
\frac{g^{\mathrm{E}}}{R T}= & \frac{P}{R T}\left(V-\sum_{i} x_{i} V_{i}\right)-\left[\ln \frac{P(V-b)}{R T}\right. \\
& \left.-\sum_{i} x_{i} \ln \frac{P\left(V_{i}-b_{i}\right)}{R T}\right]-\left[\frac{a}{R T V}-\sum_{i} x_{i} \frac{a_{i}}{R T V_{i}}\right]
\end{aligned}
$$

Equation 7 can be written in a more compact form as

$$
\frac{g^{\mathrm{E}}}{R T}=\frac{P V^{\mathrm{E}}}{R T}+\frac{A^{\mathrm{E}}}{R T}=\frac{P V^{\mathrm{E}}}{R T}-\frac{S^{\mathrm{E}}}{R}+\frac{U^{\mathrm{E}}}{R T}
$$

$$
=\frac{P V^{\mathrm{E}}}{R T}+\sum_{i} x_{i} \ln \left(\frac{V_{i}-b_{i}}{V-b}\right)+\frac{1}{R T}\left[\sum_{i} x_{i}\left(\frac{a_{i}}{V_{i}}\right)-\left(\frac{a}{V}\right)\right]
$$

The excess volume term can be easily recognized, but are the other two terms of eq $8 b$ the excess entropy and excess energy contributions, thus reflecting (to some extent) the entropic and energetic effects?

The excess entropy and excess energy contributions can be independently calculated from the residual properties as follows:

$$
\begin{aligned}
& \begin{aligned}
h^{\mathrm{E}}=h^{\text {res }}- & \sum x_{i} h_{i}^{\text {res }}
\end{aligned} \\
& \text { So that: } \begin{aligned}
h^{\mathrm{E}}= & R T(Z-1)-\frac{\left(a-T a^{\prime}\right)}{V} \\
& -\sum_{i} x_{i}\left[R T\left(Z_{i}-1\right)-\frac{\left(a_{i}-T a_{i}^{\prime}\right)}{V_{i}}\right]
\end{aligned} \\
& \text { Finally: } h^{\mathrm{E}}=P V^{\mathrm{E}}+\sum_{i} x_{i}\left(\frac{a_{i}-T a_{i}^{\prime}}{V_{i}}\right)-\left(\frac{a-T a^{\prime}}{V}\right)
\end{aligned}
$$

where $a^{\prime}=\frac{d a}{d T}$. It is thus clear that only if the energy parameter is $T$-independent does the excess enthalpy reduce to the form "implied" by eq $8 \mathrm{~b}$ :

$$
h^{\mathrm{E}}=P V^{\mathrm{E}}+\sum_{i} x_{i}\left(\frac{a_{i}}{V_{i}}\right)-\left(\frac{a}{V}\right)
$$

Similarly, for the excess entropy, we have

$$
\begin{aligned}
S^{\mathrm{E}}=S^{\text {res }}- & \sum x_{i} S_{i}^{\text {res }} \\
\text { So that } \frac{S^{\mathrm{E}}}{R}= & \ln \left(\frac{P(V-b)}{R T}\right)+\frac{a^{\prime}}{R V} \\
& -\left(\sum_{i} x_{i} \ln \frac{P\left(V_{i}-b_{i}\right)}{R T}+\frac{a_{i}^{\prime}}{R V_{i}}\right) \\
\text { Finally, } \frac{S^{\mathrm{E}}}{R}= & -\sum_{i} x_{i} \ln \left(\frac{V_{i}-b_{i}}{V-b}\right)+\frac{1}{R}\left(\frac{a^{\prime}}{V}-\sum_{i} x_{i} \frac{a_{i}^{\prime}}{V_{i}}\right)
\end{aligned}
$$

Again, only for a $T$-independent energy parameter does the excess entropy correspond to the equation implied by eq $8 \mathrm{~b}$ :

$$
\frac{S^{\mathrm{E}}}{R}=-\sum_{i} x_{i} \ln \left(\frac{V_{i}-b_{i}}{V-b}\right)
$$

Of course the excess Gibbs energy and the fugacity coefficient can be calculated, either from eqs 5 and 6, or as the sum of the excess enthalpies and entropies from eqs 9 and 11, yielding the general equations below where the excess entropy and enthalpy/energy contributions can be clearly identified: 


$$
\begin{aligned}
\frac{g^{\mathrm{E}}}{R T}= & -\frac{S^{\mathrm{E}}}{R}+\frac{h^{\mathrm{E}}}{R T} \\
= & \sum_{i} x_{i} \ln \left(\frac{V_{i}-b_{i}}{V-b}\right)-\frac{1}{R}\left(\frac{a^{\prime}}{V}-\sum_{i} x_{i} \frac{a_{i}^{\prime}}{V_{i}}\right)+\frac{P V^{\mathrm{E}}}{R T} \\
& +\frac{1}{R T}\left[\sum_{i} x_{i}\left(\frac{a_{i}-T a_{i}^{\prime}}{V_{i}}\right)-\left(\frac{a-T a^{\prime}}{V}\right)\right] \\
\ln \varphi= & \frac{g^{\text {res }}}{R T}=-\frac{S^{\text {res }}}{R}+\frac{h^{\text {res }}}{R T}=-\ln \left(\frac{P(V-b)}{R T}\right)-\frac{a^{\prime}}{R V} \\
+ & (Z-1)-\frac{1}{R T}\left(\frac{a-T a^{\prime}}{V}\right)
\end{aligned}
$$

which is of course identical to eq 6 :

$$
\ln \varphi=-\ln \left(\frac{P(V-b)}{R T}\right)+\left(\frac{P V}{R T}-1\right)-\frac{1}{R T} \frac{a}{V}
$$

As expected, eqs 13 and 14 are identical to the "simpler forms" of eqs 5 or 6 ; that is, all $T$-derivatives of energy equations cancel out in eqs 13 and 14 (as can be easily seen), however the "simple" expressions for the excess energy and entropy seen in eqs 10 and 12 can only be obtained when the energy parameter is $T$-independent.

Table S1 of the Supporting Information presents the relevant equations for the vdW and SRK EoS.

The $T$-dependency of the energy parameter in the form of the derivative of the parameter with temperature will affect both the excess entropy and energy (enthalpy) terms and, in general terms, $S^{\mathrm{E}}$ and $U^{\mathrm{E}}$ (or $H^{\mathrm{E}}$ ) should be given by eqs 11 and 9 , respectively. We also notice that the $\mathrm{d} a / \mathrm{d} T$ term is not included in the residual Helmholtz or Gibbs energies or the excess Helmholtz and Gibbs energies and naturally neither in the expression for the fugacity coefficient.

Once again, we recognize Gibbs statement that "the total is easier or simpler than the sum of its parts" in the sense that $g^{\mathrm{E}}$ is indeed much simpler than adding or calculating separately the excess enthalpy/energy and entropy contributions.

At this moment, it would be interesting to discuss some differences in the terminology used in equations of state and activity coefficient models, with regards to the energy and entropy terms. This is also useful as later we will examine what can be learned from the vdW EoS when it is written as an activity coefficient model.

Activity coefficient models are often expressed in terms of excess Gibbs energy or activity coefficient as

$$
\begin{aligned}
& \frac{g^{\mathrm{E}}}{R T}=\frac{g^{\mathrm{E}, \mathrm{comb}-F V}}{R T}+\frac{g^{\mathrm{E}, \text { res }}}{R T} \\
& \ln \gamma_{i}=\ln \gamma_{i}^{\mathrm{comb}-\mathrm{FV}}+\ln \gamma_{i}^{\text {res }}
\end{aligned}
$$

The comb-FV (combinatorial-free volume) roughly accounts for the size/shape effects and the so-called "residual" term accounts for the energetic interactions. We will, in the remainder of this manuscript, use "E,res" for the "residual" contributions to the excess Gibbs, Helmholtz energies, or entropies in order to avoid confusion with the rigorous term of residual properties as defined in thermodynamics.

For example, the universal quasichemical (UNIQUAC), UNIQUAC functional activity coefficient (UNIFAC), and Wilson models contain both combinatorial-FV and "residual" terms, while the nonrandom two-liquid (NRTL) has only a "residual" Gibbs energy, or activity coefficient term.

As indicated above, a point of caution: the "residual" part of the excess Gibbs energy or activity coefficient is not the same as the rigorous residual Gibbs energy from thermodynamics, as shown in eq 5. The use of the word "residual" in both contexts may cause some confusion. The residual properties in thermodynamics have a clear definition and connection to excess properties:

$$
\begin{aligned}
& M_{T, P, x}^{\mathrm{res}}=M_{T, P, x}-M_{T, P, x}^{\mathrm{ig}} \\
& M^{\mathrm{E}}=M^{\mathrm{res}}-\sum_{i} x_{i} M_{i}^{\mathrm{res}}
\end{aligned}
$$

The "residual" in eqs 15 and 16 probably means the "remaining" contribution to the excess Gibbs energy or activity coefficient, that is, the contributions not associated with the size/shape effects.

Since excess Gibbs energy is rigorously connected to excess entropy and excess enthalpy, the following equation can be written:

$$
\frac{g^{\mathrm{E}}}{R T}=-\frac{S^{\mathrm{E}}}{R}+\frac{h^{\mathrm{E}}}{R T}=\frac{g^{\mathrm{E}, \mathrm{comb}-\mathrm{FV}}}{R T}+\frac{g^{\mathrm{E}, \mathrm{res}}}{R T}
$$

Looking at eq 18 and the physical significance of the comb-FV and "residual" terms, it is tempting to state that the entropic effects $\left(S^{\mathrm{E}}\right)$ and comb-FV are identical and the same can be said for the enthalpic $\left(h^{\mathrm{E}}\right)$ and "residual" terms.

This is not precise. Applying the general equations below to eq 15 :

$$
\begin{aligned}
& \left(\frac{\partial g^{\mathrm{E}}}{\partial T}\right)_{P, x}=-S^{\mathrm{E}} \\
& \left(\frac{\partial\left(\frac{g^{\mathrm{E}}}{T}\right)}{\partial T}\right)_{P, x}=-\frac{h^{\mathrm{E}}}{T^{2}}
\end{aligned}
$$

We get (if the comb-FV term of the excess Gibbs energy is linearly proportional to temperature like, e.g., in the FloryHuggins expression):

$$
\begin{aligned}
& -T S^{\mathrm{E}}=g^{\mathrm{E}, \mathrm{comb}-\mathrm{FV}}-T S^{\mathrm{E}, \text { res }} \\
& h^{\mathrm{E}}=g^{\mathrm{E}, \mathrm{res}}+T S^{\mathrm{E}, \mathrm{res}}
\end{aligned}
$$

and apparently we recover eq 18 :

$$
g^{\mathrm{E}}=-T S^{\mathrm{E}}+h^{\mathrm{E}}=g^{\mathrm{E}, \mathrm{comb}-\mathrm{FV}}+g^{\mathrm{E}, \text { res }}
$$

In the above expressions, the so-called "residual" contribution to the excess entropy present in both eqs $20 \mathrm{a}$ and $20 \mathrm{~b}$ stems from the $T$-dependency of the "residual" term:

$$
S^{\mathrm{E}, \mathrm{res}}=-\left(\frac{\partial g^{\mathrm{E}, \mathrm{res}}}{\partial T}\right)_{x}
$$

From the above, it can be concluded that neither the comb-FV nor the "residual" terms of activity coefficient models are exactly equivalent to the excess entropy and excess enthalpy terms. They may be "close", but both must be corrected due to the T-dependency of the "residual" term (see eqs 20a and $20 \mathrm{~b})$. From the above, it can be understood that even NRTL 
has an excess entropy contribution (which is equal to $S^{\mathrm{E} \text {,res }}$ ), due to the $T$-dependency of its residual term. This is in contrast to what is sometimes stated that NRTL is a "purely" energetic model. ${ }^{52}$

Similar comments can be made for the vdW equation of state, if we compare eqs $8 \mathrm{~b}$ and 13 and try to identify the comb-FV and "residual" contributions to the excess Gibbs energy (or even better the excess Helmholtz energy, thus ignoring the excess volume term). By doing so, in general terms we can write for the vdW EoS:

$$
\begin{gathered}
-T S^{\mathrm{E}}=A^{\mathrm{E}, \text { comb-FV }}-T S^{\mathrm{E}, \text { res }} \quad \text { with } \\
A^{\mathrm{E}, \text { comb-FV }}=R T \sum_{i} x_{i} \ln \left(\frac{V_{i}-b_{i}}{V-b}\right) \\
U^{\mathrm{E}}=A^{\mathrm{E}, \text { res }}+T S^{\mathrm{E}, \text { res }} \\
A^{\mathrm{E}}=-T S^{\mathrm{E}}+U^{\mathrm{E}}=A^{\mathrm{E}, \text { comb-FV }}+A^{\mathrm{E}, \text { res }}
\end{gathered}
$$

Note that $A^{\mathrm{E}, \mathrm{comb}-\mathrm{FV}}$ was identified as the Helmholtz energy part stemming from the repulsive part of the vdW EoS (or, in other words, this is the excess entropy of the vdW EoS when a constant energy parameter is selected, see eq 12). Equation 23a highlights a linear $T$-dependency of $A^{\mathrm{E}, \mathrm{comb}-\mathrm{FV}}$, making it similar to a Flory-Huggins expression.

The Supporting Information shows an application of eqs $20 \mathrm{a}, 20 \mathrm{~b}$ or $23 \mathrm{a}, 23 \mathrm{~b})$ in the case of the Wilson activitycoefficient model.

Thus, once again, the reason that, in eq 24, the comb-FV and "residual" parts are not identical to the excess entropy and excess energy contributions is the presence of $S^{\mathrm{E}, \text { res }}$ which is due to the $T$-dependency of the energy parameter and can be recognized in both eqs 9 and 11 for the vdW EoS:

$$
S^{\mathrm{E}, \mathrm{res}}=\frac{a^{\prime}}{V}-\sum_{i} x_{i} \frac{a_{i}^{\prime}}{V_{i}}
$$

The presence of this $T$-dependency of the energy term is what prohibits the "complete" separability of size and energy terms in the vdW EoS (when the energy parameter is a function of temperature).

If, on the other hand, we assume that the energy parameter is $T$-independent ( $\mathrm{as}$ is often considered for $\mathrm{vdW}$ in its traditional form), then a much simpler result is obtained:

$$
\begin{aligned}
U^{\mathrm{E}} & =A^{\mathrm{E}, \mathrm{res}}=\sum_{i} x_{i}\left(\frac{a_{i}}{V_{i}}\right)-\left(\frac{a}{V}\right) \\
-T S^{\mathrm{E}} & =A^{\mathrm{E}, \mathrm{comb}-\mathrm{FV}}=R T \sum_{i} x_{i} \ln \left(\frac{V_{i}-b_{i}}{V-b}\right) \\
\frac{A^{\mathrm{E}}}{R T} & =\frac{A^{\mathrm{E}, \mathrm{comb}-\mathrm{FV}}}{R T}+\frac{A^{\mathrm{E}, \mathrm{res}}}{R T} \\
& =\sum_{i} x_{i} \ln \left(\frac{V_{i}-b_{i}}{V-b}\right)+\frac{1}{R T}\left[\sum_{i} x_{i}\left(\frac{a_{i}}{V_{i}}\right)-\left(\frac{a}{V}\right)\right]
\end{aligned}
$$

We will not discuss it in detail in this work, but it can be easily shown that similar concepts about the separability of entropic and enthalpic terms and the importance of the T-dependency of the energy parameter are valid for other cubic EoS of the vdW-type, like SRK, see the relevant equations in Table S1 of the Supporting Information.
Abbott and Prausnitz ${ }^{53}$ and Abbott ${ }^{46}$ had arrived to similar conclusions in an earlier analysis of the vdW EoS. They first indicate that a more appropriate division of the compressibility factor of vdW (or other similar EoS) is not just the sum of rep(ulsions) and attr(actions):

$$
Z=Z^{\text {rep }}+Z^{\text {attr }}
$$

They recognize that repulsions can be both "hard" for example, as represented by a hard-sphere fluid term (dominating at very high/infinite temperature) and "soft" (for other temperatures; could be accounted by an additional term or a temperaturedependent covolume):

$$
Z=Z^{\text {hard-sphere }}+Z^{\text {attr+soft repulsions }}
$$

They showed that the vdW-type EoS can be expressed in the following general form for the residual Helmholtz energy:

$$
\begin{aligned}
A^{\mathrm{res}} & =R T \int_{0}^{\rho}(Z-1) \frac{\mathrm{d} \rho}{\rho} \\
& =R T \int_{0}^{\rho}\left(Z_{0}-1\right) \frac{\mathrm{d} \rho}{\rho}+T \int_{0}^{\tau} U^{\mathrm{res}} \mathrm{d} \tau
\end{aligned}
$$

where $\rho=\frac{1}{V} \tau=\frac{1}{T} Z_{0}=\lim _{\tau \rightarrow 0} Z$ or alternatively as

$$
\frac{A^{\mathrm{res}}}{R T}=-\frac{S_{0}^{\mathrm{res}}}{R}+\frac{1}{R} \int_{0}^{\tau} U^{\mathrm{res}} \mathrm{d} \tau
$$

where $S_{0}^{\text {res }}=\lim _{\tau \rightarrow 0} S^{\text {res }}$ In eqs $28 \mathrm{a}$ and $28 \mathrm{~b}$, the term containing the $Z_{0}$ or the residual entropy at infinite $T$ is the "strictly" entropic part, or in other words, the hard-core part of the potential, while the term with $U^{\text {res }}$ includes both intermolecular interactions and a second entropic part from the soft part of the repulsive potential.

Abbott and Prausnitz ${ }^{53}$ also showed, inspired from the vdW approach and the free-volume concept, that eq $28 \mathrm{a}$ or $28 \mathrm{~b}$ can be now written using "molecular concepts" in an equivalent form as

$$
A^{\mathrm{res}}=-R T \ln \left(\frac{V_{\mathrm{f}}}{V}\right)-\tilde{a} \rho
$$

where $V_{\mathrm{f}}$ is the free volume term $(=V-b$ for the vdW EoS) and $\tilde{a}=V T \int{ }_{0}^{\tau} U^{\text {res }} \mathrm{d} \tau$ is a term called the "energy intensity" which is further expressed as

$$
\int_{0}^{\tau} U^{\text {res }} \mathrm{d} \tau=U^{\text {res }}-T\left(S^{\text {res }}-S_{0}^{\text {res }}\right)
$$

The term in parentheses in eq $29 \mathrm{~b}$ is the entropic contribution due to attractions and soft repulsions.

Applying the aforementioned concepts to the van der Waals equation of state, we can write the following equivalent expressions for the residual Helmholtz energy:

$$
\begin{aligned}
A^{\text {res }} & =-T S^{\text {res }}+U^{\text {res }} \\
A^{\text {res }} & =-R T \ln \left(\frac{V-b}{V}\right)-\frac{a}{V} \\
A^{\text {res }} & =-T S_{0}^{\text {res }}+T \int_{0}^{\tau} U^{\text {res }} \mathrm{d} \tau \\
& =\left(-T S_{0}^{\text {res }}\right)+\left\{U^{\text {res }}-T\left(S^{\text {res }}-S_{0}^{\text {res }}\right)\right\}
\end{aligned}
$$




$$
A^{\mathrm{res}}=-R T \ln \left(\frac{V-b}{V}\right)+\left\{\left(-\frac{a}{V}+\frac{a^{\prime} T}{V}\right)-T\left(\frac{a^{\prime}}{V}\right)\right\}
$$

Equations 30b, 30c, and 30d are identical but are written in somewhat different forms in order to identify the different contributions to the residual Helmholtz energy. The overall conclusions and take-home message is the same, as that given previously: we can obtain for the vdW EoS complete separability of $S^{\text {res }}$ and $U^{\text {res }}$ (entropic and energetic interactions) only if the energy parameter is $T$-independent and in the latter case $U^{\text {res }}$ is also $T$-independent.

\section{MIXING AND COMBINING RULES WITH THE VAN DER WAALS EQUATION}

3.1. The vdW 1 f Mixing Rules. Nowhere in section 2 did we need to use or imply a specific type of mixing rules, but of course these rules are needed for obtaining numerical values of the excess and all other properties mentioned there. The results of any EoS can be very much dependent on the choice of mixing and combining rules. We address this issue in this section.

Although van der Waals himself was, in his Nobel-prize speech, rather reluctant to talk about mixtures, devoting a very short part of the speech on this and no equations were shown, his name and the classical use of the vdW (and other cubic) EoS are associated with the so-called van der Waals one-fluid mixing rules with which he had frequently worked:

$$
a=\sum_{i} \sum_{j} x_{i} x_{j} a_{i j} \quad b=\sum_{i} \sum_{j} x_{i} x_{j} b_{i j}
$$

These quadratic mixing rules satisfy also the statistically mechanically imposed quadratic dependency of the second virial coefficient $\left(=b-a / R T\right.$ for vdW and SRK, $\mathrm{PR}^{54}$ but not for the third virial coefficient, see Sandler $\left.{ }^{11}\right)$. This limiting behavior for the second virial coefficient has been considered important by some (e.g., Wong and Sandler ${ }^{55}$ ), and it sometimes has even been stated ${ }^{56}$ that mixing rules which are not in agreement with this theoretical constraint lead to "thermodynamic inconsistency". This is, in our view, a rather strong statement and there is nothing thermodynamically inconsistent with models/mixing rules which do not obey the quadratic composition dependency of the second virial coefficient. Moreover, several researchers have stated that they have not seen any practical implications even if this limit of the second virial coefficient mixing rule is not fulfilled (Vidal and Bogdanic, ${ }^{57}$ Michelsen and Møllerup ${ }^{58}$ ).

The mixing rules of eq 31 are typically used with the geometric mean combining rule for the cross-energy parameter, justified from London theory of intermolecular forces and the arithmetic-mean rule for the cross covolume parameter.

The van der Waals EoS has been used, in a translated form, with success by Tassios and co-workers (e.g., Czerwienski et al. $^{59}$ and Tassios ${ }^{47}$ ) for VLE calculations of nonpolar/weakly polar mixtures using these simple mixing rules, and the results are comparable to the PR equation of state. Please notice that the translation does not affect the phase equilibria calculations, and it can be shown that it does not change the activity coefficient calculated from the EoS either (i.e., the same values are obtained from the original and translated vdW for the activity coefficients) if the volume translation is given by a linear mixing rule. ${ }^{60,61}$
If we assume these classical mixing and combining rules and we ignore the excess volume contribution, the excess Gibbs energy from vdW EoS from eq $8 \mathrm{~b}$ can be written (for a binary system $i-j)$ as

$$
\frac{g^{\mathrm{E}}}{R T}=\sum_{i} x_{i} \ln \frac{\varphi_{i}^{f v}}{x_{i}}+\frac{V}{R T} \varphi_{i} \varphi_{j}\left(\frac{\sqrt{a_{i}}}{V_{i}}-\frac{\sqrt{a_{j}}}{V_{j}}\right)^{2}
$$

where the free-volume and volume fractions are defined as

$$
\varphi_{i}^{\mathrm{fv}}=\frac{x_{i}\left(V_{i}-b_{i}\right)}{\sum_{j} x_{j}\left(V_{j}-b_{j}\right)} ; \quad \varphi_{i}=\frac{x_{i} V_{i}}{\sum_{j} x_{j} V_{j}}
$$

Similarly, the activity coefficient can be derived from the ratio of the fugacity of component $i$ in a mixture divided by that of a pure component as (in eq $33 \mathrm{~b}$ we show to illustrate the expression for the infinite dilution activity coefficient in a binary mixture from the vdW EoS):

$$
\begin{aligned}
\ln \gamma_{i}= & \ln \gamma_{i}^{\mathrm{comb}-\mathrm{fv}}+\ln \gamma_{i}^{\mathrm{res}}=\left(\ln \frac{\varphi_{i}^{\mathrm{fv}}}{x_{i}}+1-\frac{\varphi_{i}^{\mathrm{fv}}}{x_{i}}\right) \\
+ & \left(\frac{V_{i}}{R T}\left(\delta_{i}-\delta_{j}\right)^{2} \varphi_{j}^{2}\right) \quad \text { where } \delta_{i}=\frac{\sqrt{a_{i}}}{V_{i}} \\
\ln \gamma_{1}^{\infty}=\ln \gamma_{1}^{\mathrm{comb}-\mathrm{fv}}+\ln \gamma_{1}^{\mathrm{res}} & \ln \left(\frac{V_{1}-b_{1}}{V_{2}-b_{2}}\right)+1-\left(\frac{V_{1}-b_{1}}{V_{2}-b_{2}}\right)+\frac{V_{1}}{R T} \\
& \left(\frac{\sqrt{a_{1}}}{V_{1}}-\frac{\sqrt{a_{2}}}{V_{2}}\right)^{2}
\end{aligned}
$$

Equations 32 and 33 yield some interesting conclusions. First, we see a complete separability of the combinatorial-free volume and "residual" interactions, as in the excess Gibbs energy expression. As previously mentioned, this is not identical to a complete separability of entropic and enthalpic/energetic contributions (in terms of excess entropy and enthalpy properties). The latter is the case only if the energy parameter is $T$-independent.

The second interesting conclusion is the direct similarity of eqs $33 \mathrm{a}$ and $33 \mathrm{~b}$ with the Flory-Huggins model (here in form of free-volume rather than volume or segment fractions) supplemented by a regular solution-type term, with the "solubility parameter" from vdW defined as shown in eq 33 a. This may suggest that vdW could be used even for polymer solutions. This has been indeed done with success, and for both the vdW and other cubic EoS (Sako et al., ${ }^{62}$ Kontogeorgis et al.; ${ }^{63}$ for a review, Kontogeorgis and Folas ${ }^{32}$ ). This is further discussed later.

3.2. Corrections to Quadratic Mixing Rules. Equations $33 \mathrm{a}$ and $33 \mathrm{~b}$ are derived under the assumption of the vdW If mixing rules (eq 31) and the conventional combining rules, shown below, but without the use of any adjustable parameters:

$$
\begin{aligned}
& a_{i j}=\sqrt{a_{i} a_{j}}\left(1-k_{i j}\right) \\
& b_{i j}=\frac{b_{i}+b_{j}}{2}\left(1-l_{i j}\right)
\end{aligned}
$$

that is, the $k_{i j}$ and $l_{i j}$ shown in eqs $34 \mathrm{a}$ and $34 \mathrm{~b}$ are set to zero. 
In the more general form where we allow for interaction parameters or even other combining rules for the cross energy and cross covolume parameters, the more general expression for the activity coefficient of vdW is shown below, in eq $35 \mathrm{~b}$ for the infinite dilution activity coefficient in a binary mixture.

$$
\begin{aligned}
\ln \gamma_{i}= & \ln \gamma_{i}^{\text {comb-FV }}+\ln \gamma_{i}^{\text {res }} \\
= & \ln \left(\frac{V_{i}-b_{i}}{V-b}\right)+1-\left[\frac{V_{i}-\left(2 b_{i j}-b_{j}\right)}{V-b}\right] \\
& +\left\{\frac{a_{i}}{V_{i} R T}+\frac{a V_{i}}{V^{2} R T}-2 \frac{\sum x_{i} a_{j i}}{V R T}\right\} \\
\ln \gamma_{1}^{\infty}= & \ln \gamma_{1}^{\text {comb-FV }}+\ln \gamma_{1}^{\text {res }} \\
= & \ln \left(\frac{V_{1}-b_{1}}{V_{2}-b_{2}}\right)+1-\left[\frac{V_{1}-\left(2 b_{12}-b_{2}\right)}{V_{2}-b_{2}}\right] \\
& +\left\{\frac{a_{1}}{V_{1} R T}+\frac{a_{2} V_{1}}{V_{2}^{2} R T}-\frac{2 a_{12}}{V_{2} R T}\right\}
\end{aligned}
$$

If, in eq $35 b$, we employ the classical combining rules shown in eqs $34 \mathrm{a}$ and $34 \mathrm{~b}$ without using interaction parameters, then we arrive, after some algebra, to the simpler expressions shown in eq $33 \mathrm{~b}$. In eq $35 \mathrm{~b}$, we see a complete separability of the combining rules for the covolume and energy parameters; the former affecting only the size/comb-FV term and the latter only the energy/residual term. This is interesting, as this apparent separability is not observed in the case of the more modern cubic EoS, SRK, and PR. We will illustrate this with SRK and for simplification we show its form for the infinite dilution activity coefficient in the general case (eq 36a to compare to $35 \mathrm{~b}$ ) and when the classical combining rules are used without interaction parameters (eq $36 \mathrm{~b}$ to compare to $33 b)$ :

$$
\begin{aligned}
\ln \gamma_{1}^{\infty}= & \ln \gamma_{1}^{\text {comb-FV }}+\ln \gamma_{1}^{\text {res }} \\
= & \ln \left(\frac{V_{1}-b_{1}}{V_{2}-b_{2}}\right)+1-\left[\frac{V_{1}-\left(2 b_{12}-b_{2}\right)}{V_{2}-b_{2}}\right] \\
& +\frac{a_{1}}{b_{1} R T} \ln \left(\frac{V_{1}+b_{1}}{V_{1}}\right)+\frac{a_{2}}{b_{2} R T}\left[\frac{V_{1} b_{2}+V_{2} b_{2}-2 V_{2} b_{12}}{V_{2}^{2}+V_{2} b_{2}}\right] \\
& -\frac{a_{2}}{b_{2} R T} \ln \left(\frac{V_{2}+b_{2}}{V_{2}}\right)\left[\frac{2 a_{12}}{a_{2}}+1-\frac{2 b_{12}}{b_{2}}\right] \\
\ln \gamma_{1}^{\infty}= & \ln \gamma_{1}^{\text {comb-FV }}+\ln \gamma_{1}^{\text {res }} \\
= & \ln \left(\frac{V_{1}-b_{1}}{V_{2}-b_{2}}\right)+1-\left[\frac{V_{1}-b_{1}}{V_{2}-b_{2}}\right] \\
& +\frac{a_{1}}{b_{1} R T} \ln \frac{\left(\frac{V_{1}+b_{1}}{V_{1}}\right)}{\left(\frac{V_{2}+b_{2}}{V_{2}}\right)}+\frac{a_{2}}{b_{2} R T}\left[\frac{V_{1} b_{2}-V_{2} b_{1}}{V_{2}^{2}+V_{2} b_{2}}\right] \\
& +\frac{b_{1}}{R T} \ln \left(\frac{V_{2}+b_{2}}{V_{2}}\right)\left(\frac{\sqrt{a_{2}}}{b_{2}}-\frac{\sqrt{a_{1}}}{b_{1}}\right)^{2}
\end{aligned}
$$

As discussed elsewhere (e.g., Kontogeorgis and Economou, 2010; ${ }^{51}$ Kontogeorgis and Coutsikos, 2012 ${ }^{50}$ ), all contributions except for the last one refer to the combinatorial-free volume term (which disappears at infinite pressure, by setting $V=b)$. The last part of eqs $36 \mathrm{a}$ and $36 \mathrm{~b}$ is the energetic term which, in the simplest form, resumes a regular-solution type form, as in the vdW EoS (see eq 33a and 33b). However, while the cross energy parameter only affects the energy ("residual") term, it is evident that the combining rule for the cross covolume, $b_{12}$, affects both the combinatorial free-volume and the energy/"residual" terms or, said in another way, it appears that $b_{12}$ has a significant effect on the activity coefficient terms and (possibly also) values.

Analogous expressions can be written for the PR EoS as well. Another observation is that the "additional" to the FloryHuggins/free-volume part of the comb-FV term which appears in SRK, see eq 36b (and also a similar term in PR),

$$
+\frac{a_{1}}{b_{1} R T} \ln \frac{\left(\frac{V_{1}+b_{1}}{V_{1}}\right)}{\left(\frac{V_{2}+b_{2}}{V_{2}}\right)}+\frac{a_{2}}{b_{2} R T}\left[\frac{V_{1} b_{2}-V_{2} b_{1}}{V_{2}^{2}+V_{2} b_{2}}\right]
$$

is of importance, when activity coefficient calculations are performed (see also section 3.5, and for more details, Kontogeorgis and Economou, 2010; ${ }^{51}$ Kontogeorgis and Coutsikos, $2012^{50}$ ). This "additional" term gives the correct "size dependency" of the activity coefficients, indicating indirectly a satisfactory size/comb-FV term for the modern cubic EoS such as SRK and PR. More in section 3.5.

The importance of the combining rule for the covolume has been studied in some details by several authors. Polishuk et al. ${ }^{64,65}$ found that an EOS with classical mixing rules and proper selection of the two interaction parameters gave a relatively good quantitative agreement with experimental data of the critical line, even for systems that are difficult to correlate such as water-hydrocarbon. They highlight that while the positive $k_{12}$ values increase the extents of both VLE and LLE phase splits, the $l_{12}$ values influence the extent of the LLE, the curvatures of the bubble point lines along with the VL critical points compositions. Kontogeorgis et al. ${ }^{66}$ showed that a single $l_{i j}$ (around 0.02 ) with PR yields very good results for ethane/alkanes (from $\mathrm{C}_{12}-\mathrm{C}_{44}$ ), while a single $k_{i j}$ does not perform as well-such a $l_{i j}$ improves apparently the comb-FV term of the EoS. That the $l_{i j}$ improves the comb-FV term of the EoS for PR was also shown for alkane mixtures as well in the same study. It was also shown that the arithmetic mean rule (without corrections) performs better than other combining rules for $b_{12}$, like Lorentz and Lee and Sandler (eqs 37a and $37 \mathrm{c}$ ). The other combining rules, eqs $37 \mathrm{~b}$ and $37 \mathrm{~d}$ have been proposed by Voutsas and co-workers for the UMR-PR model ${ }^{67}$ and Gmehling and co-workers for the VTPR model. ${ }^{68}$

$$
\begin{aligned}
& b_{12}=\left(\frac{b_{1}^{1 / 3}+b_{2}^{1 / 3}}{2}\right)^{3} \\
& b_{12}=\left(\frac{b_{1}^{1 / 2}+b_{2}^{1 / 2}}{2}\right)^{2} \\
& b_{12}=\left(\frac{b_{1}^{2 / 3}+b_{2}^{2 / 3}}{2}\right)^{3 / 2} \\
& b_{12}=\left(\frac{b_{1}^{3 / 4}+b_{2}^{3 / 4}}{2}\right)^{4 / 3}
\end{aligned}
$$


Even though, the simplest combining rule performed best for cubic EoS when used with the traditional (and rather problematic, see later) mixing/combining rules for the energy parameters (as shown by Kontogeorgis et al., $1998^{66}$ ), this may not be the case when a more advanced mixing rule, such as those discussed below (in section 3.5), are considered. It would be interesting to see activity coefficient calculations with the more advanced models and these alternative combining rules for cross covolume (eqs $37 a-37 d$ ). The fact that so many rules have been proposed and no definite conclusions are made indicates that the topic merits further investigation. Activity coefficient calculations and the aforementioned separate analysis of the size/comb-FV and energy/"residual" terms can contribute to a clarification of the situation.

3.3. Predicting the Interaction Parameters for the Quadratic Mixing Rules. The quadratic mixing rules, eq 31, are proposed for the vdW (and other cubic EoS) but they have a theoretical foundation on the conformal solution theory and the one-fluid approach as proposed in literature several years after van der Waals from Leland and co-workers (see also the review by Gubbins, $2013^{14}$ ). They have showed that these mixing rules can be derived more rigorously from molecular considerations and expressed in a more general way in terms of the microscopic molecular parameters, energy, and diameter, as follows:

$$
\begin{aligned}
& \varepsilon \sigma^{3}=\sum_{i} \sum_{i} x_{i} x_{j} \varepsilon_{i j} \sigma_{i j}^{3} \\
& \sigma^{3}=\sum_{i} \sum_{i} x_{i} x_{j} \sigma_{i j}^{3}
\end{aligned}
$$

Gubbins (2013) ${ }^{14}$ mentioned that these vdW 1f mixing rules were shown, when compared to molecular simulation studies, to perform much better for size asymmetric systems compared to other types of random-mixing type rules previously proposed by Longuet-Higgins but also better compared to $n$ fluid theories proposed later.

These mixing rules do not assume a specific combining rule for the cross parameters, but the usual cases are the Lorentz (for the cross covolume) and Berthelot (for the cross energy) combining rules:

$$
\begin{aligned}
& \varepsilon_{12}=\sqrt{\varepsilon_{1} \varepsilon_{2}} \\
& \sigma_{12}=\frac{\sigma_{1}+\sigma_{2}}{2}
\end{aligned}
$$

Sometimes, see Gubbins (2013), ${ }^{14}$ we notice in the literature that the Lorentz-Berthelot name is used not only for the combining rules of eqs $39 \mathrm{a}$ and $39 \mathrm{~b}$, but also for the "equivalent" rules for the macroscopic EoS parameters, $a, b$, eq $34 \mathrm{a}$ and $34 \mathrm{~b}$. This is, however, not correct and the equivalent combining rules to Lorentz-Berthelot are shown later in this section (eqs $44 \mathrm{a}$ and $44 \mathrm{~b}$ ).

There have been several studies on the applicability range of the conformal solutions theory (vdW 1f mixing rules, eqs $38 \mathrm{a}$ and $38 \mathrm{~b}$ also with the combining rules, eqs $39 \mathrm{a}$ and $39 \mathrm{~b}$ ) with the most convincing and general study that of Harismiadis et al. (1991). ${ }^{69}$ These authors show that, contrary to what was previously thought, the vdW if theory and molecular simulation agree well in their predictions of phase coexistence envelopes (especially for pressure-composition curves, less so for density-pressure ones), even for highly asymmetric mixtures with components that differ in volume by a factor of 8 . Their results establish the validity of conformal solutions theory for simple mixtures of much greater degree of asymmetry than previous investigations. The authors admit that, while the vdW if theory can give good estimates for the coexistent properties of highly asymmetric mixtures, it may not correctly predict the chemical potentials at infinite dilution or the excess properties of these mixtures. These may also depend on the values of the combining rules as well.

Gubbins in his review $(2013)^{14}$ writes that the Lorentz combining rule is closer to the reality, while the weakest of the two combining rules is the Berthelot one for the molecular cross energy parameter. We agree on the second remark as, exactly as Gubbins writes, in eq 39a should be expected corrections due to size and ionization potential differences.

Indeed, starting from the London theory of intermolecular forces, the cross intermolecular potential is related to the selfpotentials and the ionization potentials $I_{i}$ :

$$
\Gamma_{12}=\sqrt{\Gamma_{1} \Gamma_{2}}\left(\frac{2 \sqrt{I_{1} I_{2}}}{I_{1}+I_{2}}\right)
$$

This equation can be enhanced further if we assume an expression for the intermolecular potential. Hudson and McCoubrey $(1960)^{70}$ used the Lennard-Jones potential. If we use more generally the Mie potential with an attractive exponent $n$, then the final equation is (recognizing in this way the comment of Gubbins on the range of applicability of the Berthelot rule):

$$
\varepsilon_{12}=\sqrt{\varepsilon_{1} \varepsilon_{2}}\left(\frac{\sqrt{\sigma_{1} \sigma_{2}}}{\sigma_{12}}\right)^{n}\left(\frac{2 \sqrt{I_{1} I_{2}}}{I_{1}+I_{2}}\right)
$$

It is thus clear that according to the theory of London/ Hudson-McCoubrey, the terms with the last two parentheses in eq 41 are a type of "correction" to the Berthelot rule and could be used to calculate a value of the interaction parameter (e.g., in molecular models like SAFT which indeed are based on molecular energies and diameters).

For the vdW and other cubic EoS, there is an important step prior to obtaining an expression for the interaction parameter. We use the proportionalities which are deducted by the comparison of the vdW If mixing rules in the molecular (eqs $38 \mathrm{a}$ and $38 \mathrm{~b}$ ) and macroscopic parameters (eq 31):

$$
\varepsilon \propto \frac{a}{b} \quad \sigma^{3} \propto b
$$

The final result is (we substitute eqs 42 in eq 41 ):

$$
a_{12}=\sqrt{a_{1} a_{2}}\left(\frac{\sqrt{b_{1} b_{2}}}{b_{12}}\right)^{n / 3-1}\left(\frac{2 \sqrt{I_{1} I_{2}}}{I_{1}+I_{2}}\right)
$$

which can be simplified if we accept the relationship between the ionization potential term and the covolumes presented by Coutinho et al. $(1994,2000):^{71,72}$

$$
a_{12}=\sqrt{a_{1} a_{2}}\left(\frac{\sqrt{b_{1} b_{2}}}{b_{12}}\right)^{n / 3-2}
$$

It is of interest to note that the geometric-mean combining rule for the cross-energy parameter is obtained from eq $43 \mathrm{~b}$ with the exponent $n=6$ (Lennard-Jones value).

The use of eq $43 \mathrm{~b}$ leads to the following equation for the interaction parameter: 


$$
k_{12}=1-\left(\frac{\sqrt{b_{1} b_{2}}}{b_{12}}\right)^{n / 3-2}
$$

These methods to estimate $k_{i j}$, eq $43 \mathrm{c}$ and the rule which can be deduced from eq 41, appear useful and predictive, but their applicability has been limited and with rather mixed results. Equation 41 -based $k_{i j}$ has been used by Tihic for PC-SAFT ${ }^{73}$ and in a similar form adapted for SAFT-VR by Haslam et al. ${ }^{74}$ In the case of PC-SAFT, the predicted $k_{i j}$ values (using $n=6$ ) are for various gas-alkanes only in qualitative agreement with the "optimum" $k_{i j}$ values regressed from experimental data (and even poorer for polar molecules). These calculations have several uncertainties; for example, ionization potential values are not available for heavy alkanes.

Equation $43 \mathrm{~b}$ has been used for SRK by Coutinho et al. $(1994,2000)^{71,72}$ using the $n$ - exponent as fitting parameter. There are some promising results for $\mathrm{CO}_{2}$ /hydrocarbons and in the 2000 manuscript it is shown that for various series of systems (diverse gas/alkanes, etc.) the $n$-exponent decreases with size asymmetry, while $k_{i j}$ sometimes decreases and sometimes increases. Thus, the combining rule from Hudson-McCoubrey, ${ }^{70}$ shown in eq $43 \mathrm{~b}$, has some merit at least for qualitative estimations and for predicting some trends of the interaction parameter. This work has not been pursued further and it remains to be seen whether this equation can, in the future, develop to a semipredictive estimation of the interaction parameter.

Finally, it is interesting to see that the Lorentz-Berthelot rule (eq 39a and 39b) can be expressed in terms of $\mathrm{vdW} /$ cubic EoS parameters as

$$
\begin{aligned}
& a_{12}=\sqrt{a_{1} a_{2}}\left(\frac{b_{12}}{\sqrt{b_{1} b_{2}}}\right) \\
& b_{12}=\left(\frac{b_{1}^{1 / 3}+b_{2}^{1 / 3}}{2}\right)^{3}
\end{aligned}
$$

These eqs $44 \mathrm{a}$ and $44 \mathrm{~b}$ cannot be used directly in the cubic EoS; the results will not be successful as shown in the literature (Kontogeorgis et al., 1998, ${ }^{66}$ etc.). Thus, the LorentzBerthelot combining rules are far less successful compared to the conventional combining rules for real fluids in the context of cubic EoS. Equation 44a has been used in connection with the extension of $\mathrm{vdW}$ to polymers (Kontogeorgis et al., $\left.1994^{63}\right)$.

However, it is appropriate to use the combining rules of eqs $44 \mathrm{a}$ and $44 \mathrm{~b}$ in cubic EoS when we wish to compare their results against Lennard-Jones mixture simulation data, which are also generated using the Lorentz-Berthelot combining rules. This is a way to investigate in a systematic and unbiased way whether there are any inherent deficiencies of cubic EoS that do not permit their applications to asymmetric mixtures. This analysis has been done by Harismiadis et al. (1994) ${ }^{75}$ and the predictive results of this study (no interaction parameters are used) show that, at least for pressure-composition phase diagrams, cubic EoS can be used (together with vdW If mixing rules and Lorentz-Berthelot combining rules) to model mixtures of significantly different size and energy compounds. It appears, thus, that cubic EoS do not have inherent limitations in describing phase equilibria for asymmetric systems, while problems may occur for densities and excess properties.

Equations $38 \mathrm{a}$ and $38 \mathrm{~b}$ have been generalized with leaving some or all of the exponents freely defined, resulting to a large number of mixing rules, either for the molecular or for the $\mathrm{vdW} /$ cubic EoS parameters. Some have found applications in specific cubic EoS for specialized applications; none of these generalizations has received any wide acceptance. We will not pursue them further here but a review of these diverse combining rules can be found elsewhere (Kontogeorgis and Folas, $2010^{32}$ ).

At this step, let us also mention that predictive methods for estimating $k_{i j}$ derived from the combination of an EoS $/ \mathrm{g}^{\mathrm{E}}$-type mixing rule with a predictive method for estimating $\mathrm{g}^{\mathrm{E}}$ parameters (e.g., the PPR78 approach) are presented in section 3.5.

To our knowledge, there are no successful predictive tools for predicting the $l_{i j}$ parameter (irrespectively of the combining rule used). When cubic EoS such as SRK and PR are used with both $k_{i j}$ and $l_{i j}$, the successful correlation results often seen ${ }^{12,76}$ are obtained at the cost of parameters which often assume unrealistic and physically incorrect values. This is because, as shown from eq 36a, these interaction parameters must be highly intercorrelated, at least for the SRK and PR EoS. This may not be the case for vdW where the various terms are highly separable, but simultaneous use of $k_{i j} / l_{i j}$ has not been extensively studied with vdW (to the best of our knowledge).

3.4. Another Look at the Separability of Terms and at the Size Effects from the vdW EoS. Use of the CarnahanStarling and similar terms suitable for hard-sphere fluids together with the vdW or other cubic attractive terms have not led to superior equations of state, ${ }^{20,41}$ maybe because real fluids' repulsive contributions cannot be represented alone by hard-spheres or because cancellation of errors is easily accomplished when the vdW-type repulsive and attractive terms are combined. There is, however, another aspect; the importance of rotational and vibrational modes in real, especially polyatomic, molecules and their density dependency. This aspect and a further understanding of the separability of terms in vdW-type EoS can be obtained from Statistical Thermodynamics using the concept of the generalized van der Waals partition function (vdW p.f.). Following, among others, Vera and Prausnitz, ${ }^{10}$ Tassios, ${ }^{47}$ and Sandler, ${ }^{11}$ the generalized vdW p.f. can be written as

$$
Q=\frac{1}{N ! \Lambda^{3}} V^{N}\left(\frac{V_{f}}{V}\right)^{N} Q_{\text {rot, vib }}^{N}\left[\exp \left(-\frac{\phi}{2 k T}\right)\right]^{N}
$$

This is a simplified form of the general partition function, as the latter cannot be easily used for engineering calculations (Tassios, $1993^{47}$ ).

In eq 45 , we identify from left to right, the ideal gas term, the combinatorial $\left(V^{\mathrm{N}}\right)$, the free volume term (with $V_{\mathrm{f}}$ ), the rotational/vibrational contributions, and the energy term with the total potential energy (which is related to both the pair intermolecular potential and the radial distribution function).

The last term in eq 45 includes the effect of the potential energy, and for its estimation are needed both the intermolecular potential and the radial distribution function, that is, the position of molecules in space. ${ }^{10}$ In this way, even this so-called energetic term does include (because of the radial distribution function, which is in principle a function of 
distance, density, and temperature) an "entropic" contribution. We will come back to this aspect later.

When the partition function is known, then the equation of state (pressure equation) and the Helmholtz energy (convenient to derive an expression for the activity coefficient) can be derived from the following general equations:

$$
\begin{aligned}
& P=k T\left(\frac{\partial \ln Q}{\partial V}\right)_{T, \text { comp }} \\
& A=-k T \ln Q
\end{aligned}
$$

Applying eq $46 a$ to eq 45 , the general form of the equation of state is

$$
P=P^{\mathrm{comb}-\mathrm{FV}}+P^{\mathrm{rot}, \mathrm{vib}}+P^{\mathrm{attr}}
$$

With three distinct contributions, from the combined combinatorial-free volume, rotational/vibrational, and energetic/attractive effects (all equations with derivatives based on total volume, $N$ is the number of molecules, and $k$ being the Boltzmann constant):

$$
\begin{aligned}
& P^{\text {comb-FV }}=N k T\left(\frac{\partial \ln V_{\mathrm{f}}}{\partial V}\right)_{T, N} \\
& P^{\text {rot, vib }}=N k T\left(\frac{\partial \ln Q_{\text {rot,vib }}}{\partial V}\right)_{T, N} \\
& P^{\text {attr }}=-\frac{N}{2}\left(\frac{\partial \phi}{\partial V}\right)_{T, N}
\end{aligned}
$$

Very often eq $47 \mathrm{c}$ is ignored, assuming that the rotational/ vibrational effects only depend on temperature. Then, using eqs $47 \mathrm{~b}$ and $47 \mathrm{~d}$ alone, the vdW EoS is derived when the free volume is given by the usual equation $\left(V_{\mathrm{f}}=V-b\right)$, and the total potential energy is given as (on a molar basis) $\phi=-\frac{2 a}{V N_{\mathrm{A}}}$

There is an interesting aspect in this derivation of the van der Waals EoS, following Sandler's approach. Sandler ${ }^{11}$ shows that the vdW EoS can be derived using the square-well potential and either assuming that the radial distribution function is constant equal to one or that the radial distribution function is density independent but a function of temperature $\left(g(r, \rho=0, T)=e^{-u(r) / k T}\right)$. In the first case $(g=1)$, vdW is derived with a temperature-independent energy parameter (original formulation of $\mathrm{vdW}$ ) and as we saw previously in this case, we have a complete separation of entropic and energetic effects. In the second case (low density limit of $g$ ), again the $\mathrm{vdW} \mathrm{EoS}$ is derived, but in this case the energy parameter is temperature-dependent (exponential function of temperature). Energetic and entropic effects are not quite as easily separable in this case and this could be indeed anticipated also from the generalized vdW p.f. and the link between the total potential energy and pair potential and radial distribution function, as mentioned previously.

Returning to the generalized vdW p.f. and eq 45 , following Prigogine, ${ }^{79}$ it can be assumed that the rotational and vibrational contribution to the partition function has a part that is density dependent which thus contributes to the equation of state. This part can be given by the expression shown in eq 48a below (and $C$ is the external degrees of freedom parameter; $3 C$ is the number of external and density dependent degrees of freedom). Moreover, as also Prigogine suggested, all three contributions (combinatorial, free-volume, and rotational) can be combined, and this is shown in eq $48 \mathrm{~b}$.

$$
\begin{aligned}
& Q=\frac{1}{N ! \Lambda^{3}} V^{N}\left(\frac{V_{\mathrm{f}}}{V}\right)^{N}\left(\frac{V_{\mathrm{f}}}{V}\right)^{N(C-1)}\left[\exp \left(-\frac{\phi}{2 k T}\right)\right]^{N} \\
& Q=\frac{1}{N ! \Lambda^{3}}\left(\frac{V_{\mathrm{f}}}{V}\right)^{N C}\left[\exp \left(-\frac{\phi}{2 k T}\right)\right]^{N}
\end{aligned}
$$

With the use of the final form of the generalized vdW p.f. eq $48 \mathrm{~b}$ and eq $46 \mathrm{a}$ (together with the SRK-attractive term potential; in absolute value equal to $\left.\varphi=-\frac{2 a N}{N_{\mathrm{A}} b} \ln \left(1+\frac{N}{N_{\mathrm{A}}} \frac{b}{V}\right)\right)$, Sako, $\mathrm{Wu}$, and Prausnitz ${ }^{62}$ presented the first cubic equation of state ever proposed for polymers:

$$
P=-\frac{a}{V(V+b)}+\frac{R T}{V-b}\left(\frac{V-b+b C}{V}\right)
$$

Using also eq 48b (only the comb, FV, and rotational contributions) and eq 46b, Kontogeorgis and co-workers ${ }^{78,79}$ developed a more general "size, free volume" expression for the excess Gibbs energy and the resulting equation for the activity coefficient:

$$
\begin{aligned}
& \frac{g^{\mathrm{E}}}{R T}=\sum_{i} x_{i} \ln \frac{\varphi_{i}^{\mathrm{vol}}}{x_{i}}+\sum_{i} x_{i} C_{i} \ln \left(\frac{\varphi_{i}^{\mathrm{fv}}}{\varphi_{i}^{v o l}}\right) \\
& \ln \gamma_{i}^{\mathrm{comb}-\mathrm{Fv}}=\left(\ln \frac{\varphi_{i}^{\mathrm{fv}}}{x_{i}}+1-\frac{\varphi_{i}^{\mathrm{fv}}}{x_{i}}\right)+C_{i} \ln \left(\frac{\varphi_{i}^{\mathrm{fv}}}{\varphi_{i}^{\mathrm{vol}}}\right) \\
&+C_{\text {mix }}\left(\frac{\varphi_{i}^{\mathrm{vol}}}{x_{i}}-\frac{\varphi_{i}^{\mathrm{fv}}}{x_{i}}\right)
\end{aligned}
$$

They called it Chain-FV and using diverse ways to get the Cparameter, for example, as $r / q$ (ratio of the vdW volume over area), they obtained improved results over the Flory-Huggins and the free-volume model of Elbro et al. $^{80}$ for activity coefficients of alkanes, solid-liquid equilibrium calculations, and against molecular simulation data. When $C=1$, the model results to the same "comb-FV" term proposed by Elbro et al. ${ }^{68}$ and also implied in the van der Waals equation. There are no rigorous approaches to predict the $C$-parameter, and different (rather similar values) have been proposed in the literature in models which use this parameter, like the UNIFAC-FV model by Oishi and Prausnitz ${ }^{81}$ and the Flory equation of state.

Sako, $\mathrm{Wu}$, and Prausnitz ${ }^{62}$ (and other researchers in subsequent studies ${ }^{82}$ ) have also been successful in modeling some nonpolar polymer systems at high pressures.

None of these approaches have been pursued very much after these publications, but both studies illustrate that it is possible to improve the representation of the "size" effects within the van der Waals framework, and in a physically meaningful way while maintaining the simplicity and original spirit of the van der Waals approach, even maintaining the cubic form of the equation of state.

3.5. The EoS/GE-Type Mixing Rules. An interesting mixing rule can be obtained if we take the limit of infinite pressure and apply it to eq $8 \mathrm{~b}$. At this limit we can assume that the volume equals to the covolume. If moreover we assume that the linear mixing rule is used for the covolume parameter, we can show that 


$$
\frac{g^{\mathrm{E}, \infty}}{R T}=\frac{1}{R T}\left(\sum_{i} x_{i} \frac{a_{i}}{b_{i}}-\frac{a}{b}\right)
$$

This is the Huron-Vidal mixing rule ${ }^{83}$ applied to the vdW EoS. We can see that this infinite pressure limit only stems from the "residual" term of the $g^{\mathrm{E}}$ expression of the EoS and the combinatorial-free volume part disappears.

Exactly the same expression can be obtained for the excess Helmholtz energy, but in this case no assumption is made for the mixing rule for the covolume parameter. As Wong and Sandler ${ }^{55}$ showed, this has the advantage that any mixing rule can be used for the covolume. Wong and Sandler ${ }^{55}$ used this extra degree of freedom to satisfy the quadratic composition dependency for the second virial coefficient.

$$
\frac{A^{\mathrm{E}, \infty}}{R T}=\frac{1}{R T}\left(\sum_{i} x_{i} \frac{a_{i}}{b_{i}}-\frac{a}{b}\right)
$$

Equation 51, when the vdW 1f mixing rules are used from section 3.1, is the van Laar equation in its predictive form. For a binary system $i-j$, one has

$$
\frac{g^{\mathrm{E}}}{R T}=\frac{1}{R T}\left(\sum_{i} x_{i} \frac{a_{i}}{b_{i}}-\frac{a}{b}\right)=\frac{b}{R T} \Phi_{i} \Phi_{j}\left(\delta_{i}-\delta_{j}\right)^{2}
$$

Where: $\Phi_{i}=\frac{x_{i} b_{i}}{\sum_{j} x_{j} b_{j}}$ and $\delta_{i}=\frac{\sqrt{a_{i}}}{b_{i}}$. The following shows the activity coefficient expression for a binary system $i-j$ :

$$
\ln \gamma_{i}=\left[\frac{b_{i}}{R T}\left(\delta_{i}-\delta_{j}\right)^{2} \Phi_{j}^{2}\right]
$$

The use of a binary interaction parameter $k_{i j}$ in the vdW If mixing rules, that is,

$$
a=\sum_{i} \sum_{j} x_{i} x_{j} \sqrt{a_{i} a_{j}}\left(1-k_{i j}\right) \text { and } b=\sum_{i} x_{i} b_{i}
$$

modifies the vdW $g^{\mathrm{E}}$ expression as follows:

$$
\begin{aligned}
\frac{g^{\mathrm{E}}}{R T} & =\frac{1}{R T}\left(\sum x_{i} \frac{a_{i}}{b_{i}}-\frac{a}{b}\right) \\
& =\frac{1}{R T}\left(\sum x_{i} \cdot \frac{a_{i}}{b_{i}}-\frac{\sum \sum x_{i} \cdot x_{j} \sqrt{a_{i} \cdot a_{j}}\left(1-k_{i j}\right)}{b}\right)
\end{aligned}
$$

After some algebra, one obtains (for a binary system $i-j$ ):

$$
\begin{aligned}
\frac{g^{\mathrm{E}}}{R T} & =\frac{x_{i} x_{j} \cdot b_{i} b_{j}}{b R T}\left[\frac{a_{i}}{b_{i}^{2}}+\frac{a_{j}}{b_{j}^{2}}-2 \frac{\sqrt{a_{i}}}{b_{i}} \frac{\sqrt{a_{j}}}{b_{j}}\left(1-k_{i j}\right)\right] \\
& =\frac{b}{R T} \Phi_{i} \Phi_{j}\left[\delta_{i}^{2}+\delta_{j}^{2}-2 \delta_{i} \delta_{j}\left(1-k_{i j}\right)\right]
\end{aligned}
$$

The $E_{i j}$ binary coefficient is now introduced:

$$
E_{i j}=\delta_{i}^{2}+\delta_{j}^{2}-2 \delta_{i} \delta_{j}\left(1-k_{i j}\right)
$$

Thus,

$$
\frac{g^{\mathrm{E}}}{R T}=\frac{b}{R T} \Phi_{i} \Phi_{j} E_{i j}
$$

Equation 59 thus establishes a connection between the binary interaction parameter $E_{i j}$ of a Van Laar-type $g^{\mathrm{E}}$ model and the binary parameter $k_{i j}$ of the $\mathrm{vdW}$ if mixing rules. This connection was advantageously used by Jaubert and coworkers $^{84-88}$ to develop the PPR78 model enabling the calculation of $E_{i j}(T)$ (and thus, of $k_{i j}(T)$, see eq 58) from a group-contribution method for the PR EoS. The $E_{i j}$ parameter is predicted using the following expression:

$$
\begin{gathered}
E_{i j}(T)=-\frac{1}{2} \sum_{k=1}^{N_{\mathrm{g}}} \sum_{l=1}^{N_{\mathrm{g}}}\left(\alpha_{i k}-\alpha_{j k}\right)\left(\alpha_{i l}-\alpha_{j l}\right) \\
A_{k l} \cdot\left(\frac{298.15}{T / \mathrm{K}}\right)^{\left(B_{k l} / A_{k l}-1\right)}
\end{gathered}
$$

In eq $60, N_{\mathrm{g}}$ is the number of different groups defined by the method (for the time being, 40 groups are defined); $a_{i k}$ is the fraction of molecule $i$ occupied by group $k$ (occurrence of group $k$ in molecule $i$ divided by the total number of groups present in molecule $i$ ). $A_{k l}=A_{l k}$ and $B_{k l}=B_{l k}$ (where $k$ and $l$ are two different groups) are constant group-interaction parameters $\left(A_{k k}=B_{k k}=0\right)$.

In the classical van der Waals-van Laar concept the solubility parameter shown in eq 53 is equal to the square root of critical pressure. Unfortunately, experimental data do not agree to this, and otherwise interesting is the observation that the nonideality is proportional to the difference of the square root of the critical pressures of the components. Thus, the predictive form of the van Laar is not very useful. It is more convenient to use the van Laar as a correlative model with two adjustable parameters. Alternatively, the predictive extension of the van Laar in the form of PPR78 or the Hildebrand's regular solution theory is often highly useful, where in eqs 53 or 54, volumes are used instead of covolumes and the solubility parameters are obtained from the "definition" (square root of cohesive energy density) rather than from the van der Waals equation.

Still, all the above shown models, the vdW EoS with the classical mixing/combining rules, section 3.1, or the excess Gibbs energy versions in the form of van Laar and regular solution theory, are not very accurate for complex systems. The underlying hypothesis is that the mixing of molecules is random, which is an assumption rigorously valid for relatively nonpolar systems. Interaction parameters and other corrections should correct for the limitations of the theory.

A more useful application of eq 51 is to see it as an opportunity for a mixing rule for the energy parameter of the equation of state, when an external local-composition activity coefficient model is implemented in the mixing rule.

The so-called EoS $/ g^{\mathrm{E}}$ mixing rules have been a "hot" topic in the $90 \mathrm{~s}$ and are now considered to be established models. Their original targets were 2-fold; to extend cubic EoS to complex mixtures, by incorporating a local composition model in the mixing rules, or extend the activity coefficients to high pressures and, at a second level, develop predictive equations of state, by incorporating a UNIFAC model in the mixing rule (or using existing parameters of local composition models from databases). The latter target, which is partially achieved by the zero reference pressure assumption, is slowly being abandoned. This approach was popular at the start with models such as MHV1/MHV2 (Dahl and Michelsen ${ }^{89}$ ) and PSRK (Gmehling ${ }^{90}$ ), but it turned out that poor results are obtained for sizeasymmetric systems due to "changing in the wrong direction" the combinatorial-free volume term of the equation of state. These problems were corrected by semiempirical approaches such as LCVM (Boukouvalas et al., 1994 ${ }^{91}$ ), but it soon 
became clear (Kontogeorgis and Vlamos, 2000 ${ }^{92}$ ) that ideally the "double combinatorial" introduced by MHV1/PSRK/ MHV2 models and the like should be completely eliminated. This is what was done by Gmehling and co-workers in recent versions of PSRK and in the VTPR model. ${ }^{68}$ Moreover, the MHV1/PSRK/MHV2 models are only "approximate zero reference pressure" models as the approximations introduced essentially change the reference pressure to an approximate zero one (see Kontogeorgis and Coutsikos, ${ }^{50}$ for details).

Thus, we believe that the infinite pressure Huron-Vidal approach is the most promising way forward (the approach also used in the Wong-Sandler and VTPR models).

Indeed, as mentioned previously, and recognizing that the combinatorial-free volume term disappears at infinite pressure, eq 51 can be rewritten as follows:

$$
\frac{g^{\mathrm{E}, \infty}}{R T}=\frac{1}{R T}\left(\sum_{i} x_{i} \frac{a_{i}}{b_{i}}-\frac{a}{b}\right)=\frac{g^{\mathrm{E}, \mathrm{res}, M}}{R T}
$$

According to eq 61, which is the general formulation of the $\mathrm{EoS} / \mathrm{g}^{\mathrm{E}}$ mixing rule (here applied at the infinite reference pressure), the vdW EoS contribution at infinite pressure can be equated to the "residual" part of an external activity coefficient $/ g^{\mathrm{E}}$ model indicated with $M$ in eq 61 for example, NRTL (which as mentioned only has a "residual" term) or the "residual" part of UNIQUAC, Wilson, or other models.

This "detail", that in principle only a "residual" term of an external activity coefficient model should be used in eq 61, when the infinite pressure limit is used, has not been clear from the start. Soave has indicated in several publications between 1993 and $2010^{93-96}$ that this is indeed the case and he employed the "residual" term of UNIQUAC and UNIFAC in several of these studies. The explanations were rather "loose" for example, "if we use the combinatorial term also, over specified values are obtained or calculations have shown that excessive decrease of volatility is shown for asymmetric alkane mixtures", etc. These statements are, in principle, correct but from the derivation of eq 61 it is, in our view, clear that at the infinite limiting pressure, only the "residual' part of the excess Gibbs energy of the external model should be used. The combinatorial-FV contribution is covered by the EoS and moreover is eliminated at the infinite pressure limit. It should be mentioned that eventually Vidal himself (e.g. Vidal ${ }^{97}$ ) recognized that it is a "residual $g^{\mathrm{E}}$ " that should be used in eq 61. He recommended in most cases NRTL but in more recent manuscripts, he suggested also using the "residual term" of UNIQUAC or even UNIFAC if a semipredictive model should be developed.

The use of the vdW EoS with the mixing rules shown in eq 61 has been indeed done by Soave ${ }^{48}$ in 1984 who used vdW with the Huron-Vidal mixing rule, as shown in eq 61 . Soave employed NRTL and ensured that accurate vapor pressures are obtained using a $T$-dependent energy parameter with two adjustable parameters:

$$
\alpha\left(T_{\mathrm{r}}\right)=1+m\left(1-T_{\mathrm{r}}\right)+n\left(\frac{1}{T_{\mathrm{r}}}-1\right)
$$

Soave obtained excellent results for the ternary acetonechloroform-methanol system, using interaction parameters exclusively obtained from binary data. If accurate densities are needed, a Péneloux-volume translation can be used.
The EoS $/ g^{\mathrm{E}}$ mixing rule affects only the "residual"/energetic part of the activity coefficient model expression from vdW which can be now written as (using the linear mixing rule for the covolume parameter):

$$
\begin{aligned}
\ln \gamma_{i} & =\ln \gamma_{i}^{\mathrm{comb}-\mathrm{FV}}+\ln \gamma_{i}^{\mathrm{res}} \\
& =\left(\ln \frac{\varphi_{i}^{\mathrm{fv}}}{x_{i}}+1-\frac{\varphi_{i}^{\mathrm{fv}}}{x_{i}}\right)+\ln \gamma_{i}^{\mathrm{res}, M}
\end{aligned}
$$

Equations 33a and 63 can be directly compared, and it can be seen that the comb-FV term is the same, while the "residual" term is now given by the external activity coefficient model, which as mentioned should only contain a "residual" term, for example, NRTL. With such an approach the regular solution term which is a direct outcome of the use of the vdW If mixing rules is replaced by the much more correct local-composition concept. As such local-composition models, at least approximately, account for (rather) strong polar interactions, their incorporation in an EoS concept permit (i) their use at high pressures and (ii) potentially being applicable for a wider range of systems, as a combinatorial-FV term is added (in NRTL).

While the comb-FV term of eq 63 is an improved FloryHuggins term (yielding reasonably accurate values of activity coefficients if experimental densities and physically correct hard-core volumes are used), this may not be the case when the volumes and covolumes are calculated from the equation of state. Even for PR, we have shown in a previous study (Kontogeorgis et al., $1998^{66}$ ) that this term yields qualitatively correct activity coefficients for athermal alkane systems (showing the expected negative deviations from Raoult's law) but still much higher than the experimental values.

Thus, while Soave ${ }^{48}$ wonders why the vdW EoS has not been used even more than the SRK, having a more rigorous theoretical basis, there may be an additional reason for the widespread use of SRK and PR.

As we have shown in previous studies ${ }^{32,50,51}$ (Kontogeorgis and Folas, 2010; Kontogeorgis and Coutsikos, 2012; Kontogeorgis and Economou, 2010) the use of SRK or PR with the Huron-Vidal mixing rules, while leaving essentially unchanged the "residual" term of eq 63, adds an additional contribution to the combinatorial-FV term of the model, stemming apparently from the attractive term (eq 36c). For SRK and at infinite dilution, the activity coefficient can be expressed as

$$
\begin{aligned}
\ln \gamma_{1}^{\infty}= & \ln \gamma_{1}^{\mathrm{comb}-\mathrm{FV}}+\ln \gamma_{1}^{\text {res }} \\
= & \ln \left(\frac{V_{1}-b_{1}}{V_{2}-b_{2}}\right)+1-\left[\frac{V_{1}-b_{1}}{V_{2}-b_{2}}\right] \\
& +\frac{a_{1}}{b_{1} R T} \ln \frac{\left(\frac{V_{1}+b_{1}}{V_{1}}\right)}{\left(\frac{V_{2}+b_{2}}{V_{2}}\right)}+\frac{a_{2}}{b_{2} R T}\left[\frac{V_{1} b_{2}-V_{2} b_{1}}{V_{2}^{2}+V_{2} b_{2}}\right] \\
& -\frac{1}{\ln 2} \ln \left(\frac{V_{2}+b_{2}}{V_{2}}\right) \ln \gamma_{1}^{\text {res }, M}
\end{aligned}
$$

Note that the EoS comb-FV term resulting from the addition of a Flory-Huggins type term with an additional term is very similar to combinatorial terms present in UNIQUAC/ UNIFAC $g^{\mathrm{E}}$ models associating a Flory-Huggins type term with a Staverman-Guggenheim contribution. 
This additional term yields a comb-FV term for SRK and PR which is in very good agreement with experimental data for the activity coefficients of several mixtures of hydrocarbons, even at high asymmetries. A typical example is shown in Figure 3.

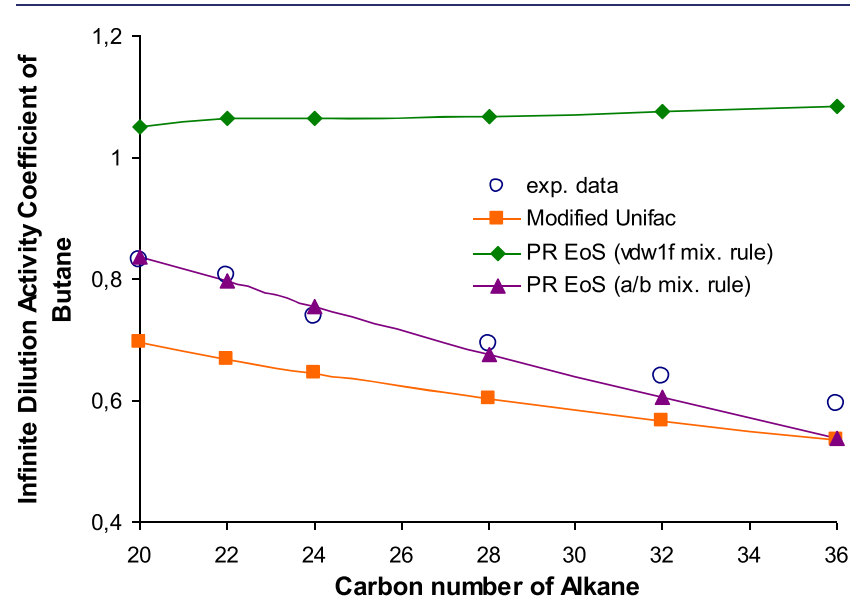

Figure 3. Activity coefficients at infinite dilution of $n$-butane in alkane solvents at $373 \mathrm{~K}$ as a function of the alkane carbon number using the Peng-Robinson equation of state. Results are shown using the vdw if mixing rules (with $k_{i j}=0$ ) and the $a / b$ mixing rule (which is essentially the Huron-Vidal mixing rule without the term for "residual" interactions; $g^{\mathrm{E}}=0$ ). For comparison are shown the results with the modified UNIFAC activity coefficient model. Reprinted with permission from ref 32. Copyright 2010 John Wiley and Sons.

Please notice that exactly the same form of PR is used (green and purple lines) with the same values of pure compound parameters and linear mixing rule for the covolume. The only difference is the mixing rule for the energy parameter, vdW if and the $a / b$ - mixing rule (eq 51 or eq 61 with $g^{\mathrm{E}}$ equal to zero).

This has been further emphasized by Sacomani and Brignole $^{98}$ (2003) who recommended this term (the combinatorial-free volume part of SRK with experimental densities), over Flory-Huggins, for polymer solutions (see Kontogeorgis and Coutsikos, $2005^{99}$ for a further discussion). The addition of the "residual" term of a local-composition model such as the NRTL renders SRK or PR/Huron-Vidal/ NRTL to be a very powerful model with excellent VLE and LLE results for a wide range of highly polar and even hydrogen bonding systems such as alcohol-hydrocarbons and waterhydrocarbons and very good predictions even for LLE/VLLE of water-alcohols/glycols-hydrocarbons over a wide temperature and pressure range (Kontogeorgis and Folas, 2010; ${ }^{32}$ Folas et al., $\left.2007^{100}\right)$.

Others (Zhong and Masuoka ${ }^{101,102}$ ) have used the same concept which yields the excellent comb-FV term for SRK and $\mathrm{PR}$ (i.e., the $a / b$ - mixing rule, eq 51 with $g^{\mathrm{E}}=0$ ) together with a mixing rule satisfying the second virial coefficient and extended cubic EoS to polymer solutions with success.

This success of the Huron-Vidal approach combined with cubic equations of state for size-asymmetric systems was not evident to Vidal himself. In one of his last manuscripts (Vidal and Bogdanic ${ }^{57}$ ) and in his book, ${ }^{103}$ Vidal stated that one of the two problems he saw with existing approaches was the description of mixtures with molecules differing in size, for example, methane with heavy alkanes. (The other was VLLE for hydrogen-bonding systems). However, we know today that the cubic EoS/Huron-Vidal approach can very accurately describe VLE and infinite dilution activity coefficients for such asymmetric systems ${ }^{32,50,51}$ (see Kontogeorgis and Folas, 2010; Kontogeorgis and Coutsikos, 2012; Kontogeorgis and Economou, 2010).

A more recent development which has the ambition of resulting to a predictive model is the already mentioned VTPR EoS from the group of professor Gmehling, ${ }^{68}$ essentially a combination of the PR/Huron-Vidal with the residual term of UNIFAC with all new UNIFAC parameters being re-estimated in the context of the EoS model and when the combinatorial term only stems from the cubic EoS.

We can thus safely state that in the form of SRK or PR, the Huron-Vidal mixing rules and a model such as the NRTL use the vdW concept in a highly satisfactory way, resulting under many circumstances in a model as accurate as some of the most successful modern association models such as CPA and PC-SAFT, at least for phase equilibrium calculations.

\section{CONCLUSIONS AND FUTURE OUTLOOK}

We have emphasized in this work that cubic equations of state, starting from van der Waals, are both of theoretical and practical merit. Their conformance with the corresponding states principle, their ability to predict most universal phase diagrams, and their approximate representation of entropic/ free-volume and energetic/enthalpic effects are some of the successes. The mixing and combining rules of $\mathrm{vdW}$ have a theoretical basis and are still used, but the true value of vdW and other EoS is when they are combined with a localcomposition model using the infinite pressure (Huron-Vidal) mixing rule. This transformation makes the cubic EoS highly useful even for polar/complex mixtures. And this has been recognized also by Soave (2002 talk at IVC-SEP meeting) who recommended to abandon the quadratic mixing rules and use Huron-Vidal instead with a suitable modified form of NRTL.

On this basis, it is not surprising that Tsonopoulos and Heidman (from Exxon) stated in $1986^{104}$ that they were satisfied with the performance of cubic EoS for petroleum applications, repeated later by Jack Heidman when he said that "Cubics are here to stay" (IVC-SEP Discussion Meeting, 1998), a view expressed also by Jose Valderrama $(2003)^{42}$ in his excellent review on "The state of the Cubic Equations of State". More recently, Hendriks $(2011)^{105}$ mentioned that the industry will first of all use proven models such as the cubic EoS and return to more advanced models only when "true gaps exist". Michael Michelsen has often been heard to say about cubic equations of state as being "a technology that refuses to die".

The capabilities of cubic EoS especially with the advanced mixing rules mentioned are unprecedented and are widely recognized by those who have carefully looked into the subject. Shortly before he passed away, Marco Satyro wrote to one of the authors of this manuscript:

"There are simple fixes we can do to the cubic estimation framework that still allow us to use it reliably to model asymmetric systems" and "I have not seen yet a detailed study showing an objective comparison between a cubic EoS and SAFT if both equations of state are parametrized in the same way".

Especially the latter statement is very significant as the model parametrization is of huge importance, as also shown in this work. The work with noncubic/advanced EoS should of course continue, but it is useful to have the above statements in mind.

Cubic EoS can, especially with the advanced mixing rules, perform several of the "basic tasks for chemical engineering", as 
called them by Zeck, ${ }^{106}$ for example, VLE and LLE with the same adjustable parameters.

Of course, there are various opinions, for example, Gupta and Olson $(2003)^{107}$ in a seminal paper wrote:

"Most past work focused on extensions of cubic vdW-type EoS... This has occurred despite the now famous recommendation of Henderson. No matter how sophisticated a mixing rule, the use of van der Waals-type cubic equations of state force their inherent limitations on the users. These are the ability to reasonably predict only the vapour pressure of a select series of components and only an approximate modelling of the effect of liquid density and compressibility. van der Waals-type cubic equations are unable to accurately model other liquid phase properties, for example, enthalpy and heat capacity also phase equilibria at high pressures, particularly the mixture critical locus."

While we do not agree with everything in the above statement, especially the comment on the mixing rules, we certainly agree with the last comment on the importance of validating all equations of state for thermal properties such as enthalpy and heat capacity. This may very well be a serious limitation of the cubic EoS, but it remains to be seen how accurate other models are. To the best of the authors' knowledge, there has been no systematic comparison or evaluation of any SAFT approach for enthalpies or heat capacities. These properties are largely ignored by the scientific community and even the modeling of other derivative properties such as speed of sound has attracted interest by researchers only rather recently.

New trends in the field such as the analysis and criteria to determine the correct temperature dependency of the energy parameter of cubic EoS (by Jaubert and co-workers ${ }^{108-111}$ ) and the use of heat capacity in the determination of EoS parameters (by Coutinho and co-workers ${ }^{112-114}$ ) may turn out to be useful approaches for the further validation not only of cubic equations of state but also of the more advanced models.

\section{ASSOCIATED CONTENT}

\section{S Supporting Information}

The Supporting Information is available free of charge on the ACS Publications website at DOI: 10.1021/acs.jced.9b00264.

Excess properties from some cubic EoS or from the Wilson equation (PDF)

\section{AUTHOR INFORMATION}

\section{Corresponding Authors}

*E-mail: gk@kt.dtu.dk.

*E-mail: romain.privat@univ-lorraine.fr.

*E-mail: jean-noel.jaubert@univ-lorraine.fr.

\section{ORCID}

Romain Privat: 0000-0001-6174-9160

Jean-Noël Jaubert: 0000-0001-7831-5684

\section{Notes}

The authors declare no competing financial interest.

\section{REFERENCES}

(1) Van der Waals, J. D. On the Continuity of the Gaseous and Liquid States; Leiden, 1873.

(2) Valderrama, J. O. The Legacy of Johannes Diderik van Der Waals, a Hundred Years after His Nobel Prize for Physics. J. Supercrit. Fluids 2010, 55 (2), 415-420.

(3) Van der Waals, J. D. The Equation of State for Gases and Liquids (December 12, 1910). In Nobel lectures including presentation speeches and laureates' biographies. Physics, 1901-1921; Amsterdam Elsevier, 1967; pp 254-265.

(4) Andrews, T., XVIII The Bakerian Lecture.-On the Continuity of the Gaseous and Liquid States of Matter. Philos. Trans. R. Soc. London 1869, 159, 575-590.

(5) Klein, M. J. The Historical Origins of the Van Der Waals Equation. Physica 1974, 73 (1), 28-47.

(6) Lebowitz, J. L.; Waisman, E. M. Statistical Mechanics of Simple Fluids: Beyond van Der Waals. Phys. Today 1980, 33 (3), 24-30.

(7) Van Konynenburg, P. H.; Scott, R. L. Critical Lines and Phase Equilibria in Binary Van Der Waals Mixtures. Philos. Trans. R. Soc., A 1980, 298 (1442), 495-540.

(8) Privat, R.; Jaubert, J.-N. Classification of Global Fluid-Phase Equilibrium Behaviors in Binary Systems. Chem. Eng. Res. Des. 2013, 91 (10), 1807-1839.

(9) Lebowitz, J. L. Exact Derivation of the Van Der Waals Equation. Physica 1974, 73 (1), 48-60.

(10) Vera, J. H.; Prausnitz, J. M. Generalized van Der Waals Theory for Dense Fluids. Chem. Eng. J. 1972, 3, 1-13.

(11) Sandler, S. I. An Introduction to Applied Statistical Thermodynamics; Wiley: Hoboken, NJ, 2011.

(12) Prausnitz, J. M.; Lichtenthaler, R. N.; Azevedo, E. G. de. Molecular Thermodynamics of Fluid-Phase Equilibria, 3rd ed.; PrenticeHall international series in the physical and chemical engineering sciences; Prentice Hall PTR: Upper Saddle River, N.J, 1999.

(13) van Laar, J. J. Zur Theorie Der Dampfspannungen von Binären Gemischen. Z. Phys. Chem. 1913, $83 U$ (1), 599-608.

(14) Gubbins, K. E. The Theory of Non-Electrolyte Solutions: An Historical Review. Mol. Phys. 2013, 111 (24), 3666-3697.

(15) Heidemann, R. A.; Prausnitz, J. M. A van Der Waals-Type Equation of State for Fluids with Associating Molecules. Proc. Natl. Acad. Sci. U. S. A. 1976, 73 (6), 1773-1776.

(16) Anderko, A. A Simple Equation of State Incorporating Association. Fluid Phase Equilib. 1989, 45 (1), 39-67.

(17) Anderko, A. Calculation of Vapor-Liquid Equilibria at Elevated Pressures by Means of an Equation of State Incorporating Association. Chem. Eng. Sci. 1989, 44 (3), 713-725.

(18) Carnahan, N. F.; Starling, K. E. Equation of State for Nonattracting Rigid Spheres. J. Chem. Phys. 1969, 51 (2), 635-636.

(19) Soave, G. Equilibrium Constants from a Modified RedlichKwong Equation of State. Chem. Eng. Sci. 1972, 27 (6), 1197-1203.

(20) Peng, D.-Y.; Robinson, D. B. A New Two-Constant Equation of State. Ind. Eng. Chem. Fundam. 1976, 15 (1), 59-64.

(21) Mathias, P. M.; klotz, H. C. Take a Closer Look at Thermodynamic Property Models. Chem. Eng. Prog. 1994, 90 (6), $67-75$.

(22) Carnahan, N. F.; Starling, K. E. Intermolecular Repulsions and the Equation of State for Fluids. AIChE J. 1972, 18 (6), 1184-1189.

(23) Bjørner, M. G. Thermodynamic Modeling of $\mathrm{CO}_{2}$ Mixtures; Technical University of Denmark: Denmark, 2016.

(24) Wong, J. O.; Prausnitz, J. M. Comments Concerning a Simple Equation of State of the van Der Waals Form. Chem. Eng. Commun. 1985, 37 (1-6), 41-53.

(25) Prausnitz, J. M. Equations of State from van Der Waals Theory: The Legacy of Otto Redlich. Fluid Phase Equilib. 1985, 24 (1-2), $63-76$.

(26) Martin, J. J. Equations of State-Applied Thermodynamics Symposium. Ind. Eng. Chem. 1967, 59 (12), 34-52.

(27) Wilczek-Vera, G.; Vera, J. H. Understanding Cubic Equations of State: A Search for the Hidden Clues of Their Success. AIChE J. 2015, 61 (9), 2824-2831.

(28) Pina-Martinez, A.; Privat, R.; Jaubert, J.-N.; Peng, D.-Y. Updated Versions of the Generalized Soave $\alpha$-Function Suitable for the Redlich-Kwong and Peng-Robinson Equations of State. Fluid Phase Equilib. 2019, 485, 264-269.

(29) Bondi, A. A. Physical Properties of Molecular Crystals, Liquids and Glasses; Wiley: New York, NY, 1968. 
(30) Elbro, H. S.; Fredenslund, Aa.; Rasmussen, P. Prediction of the Critical Volume from the van Der Waals Volume. AIChE J. 1991, 37 (7), 1107-1108.

(31) Wilson, G. M. Interpretations of Trouton's Law in Relation to Equation of State Properties. In Equations of State; Chao, K. C., Robinson, R. L., Eds.; American Chemical Society: Washington, DC, 1986; Vol. 300, pp 520-536. DOI: 10.1021/bk-1986-0300.ch025.

(32) Kontogeorgis, G. M.; Folas, G. K. Thermodynamic Models for Industrial Applications; John Wiley \& Sons, Ltd: Chichester, UK, 2010. DOI: $10.1002 / 9780470747537$.

(33) Ting, P. D.; Joyce, P. C.; Jog, P. K.; Chapman, W. G.; Thies, M. C. Phase Equilibrium Modeling of Mixtures of Long-Chain and ShortChain Alkanes Using Peng-Robinson and SAFT. Fluid Phase Equilib. 2003, 206 (1-2), 267-286.

(34) Voutsas, E. C.; Pappa, G. D.; Magoulas, K.; Tassios, D. P. Vapor Liquid Equilibrium Modeling of Alkane Systems with Equations of State: "Simplicity versus Complexity. Fluid Phase Equilib. 2006, 240 (2), 127-139.

(35) Tsivintzelis, I.; Ali, S.; Kontogeorgis, G. M. Modeling Phase Equilibria for Acid Gas Mixtures Using the CPA Equation of State. Part IV. Applications to Mixtures of $\mathrm{CO}_{2}$ with Alkanes. Fluid Phase Equilib. 2015, 397, 1-17.

(36) Tsivintzelis, I.; Ali, S.; Kontogeorgis, G. M. Modeling Systems Relevant to the Biodiesel Production Using the CPA Equation of State. Fluid Phase Equilib. 2016, 430, 75-92.

(37) Tsivintzelis, I.; Ali, S.; Kontogeorgis, G. M. Modeling Phase Equilibria for Acid Gas Mixtures Using the Cubic-Plus-Association Equation of State. 3. Applications Relevant to Liquid or Supercritical $\mathrm{CO}_{2}$ Transport. J. Chem. Eng. Data 2014, 59 (10), 2955-2972.

(38) Polishuk, I. Till Which Pressures the Fluid Phase EOS Models Might Stay Reliable? J. Supercrit. Fluids 2011, 58 (2), 204-215.

(39) Deiters, U. K.; de Reuck, K. M. Guidelines for Publication of Equations of State I. Pure Fluids (Technical Report). Pure Appl. Chem. 1997, 69 (6), 1237-1250.

(40) Kalikhman, V.; Kost, D.; Polishuk, I. About the Physical Validity of Attaching the Repulsive Terms of Analytical EOS Models by Temperature Dependencies. Fluid Phase Equilib. 2010, 293 (2), 164-167.

(41) Lopez-Echeverry, J. S.; Reif-Acherman, S.; Araujo-Lopez, E. Peng-Robinson Equation of State: 40 Years through Cubics. Fluid Phase Equilib. 2017, 447, 39-71.

(42) Valderrama, J. O. The State of the Cubic Equations of State. Ind. Eng. Chem. Res. 2003, 42 (8), 1603-1618.

(43) Wei, Y. S.; Sadus, R. J. Equations of State for the Calculation of Fluid-Phase Equilibria. AIChE J. 2000, 46 (1), 169-196.

(44) Ghosh, P. Prediction of Vapor-Liquid Equilibria Using PengRobinson and Soave-Redlich-Kwong Equations of State. Chem. Eng. Technol. 1999, 22 (5), 379-399.

(45) Rowlinson, J. S. Legacy of van Der Waals. Nature 1973, 244 (5416), 414-417.

(46) Abbott, M. M. Thirteen Ways of Looking at the van Der Waals Equation. Chem. Eng. Prog. 1989, 85 (2), 25-37.

(47) Tassios, D. P. Applied Chemical Engineering Thermodynamics; Springer Berlin Heidelberg: Berlin, Heidelberg, 1993. DOI: 10.1007/ 978-3-662-01645-9..

(48) Soave, G. Improvement of the Van Der Waals Equation of State. Chem. Eng. Sci. 1984, 39 (2), 357-369.

(49) Vidal, J.; Bogdanić, G. Equations of State, Mixing Rules and Phase Equilibrium Calculations. Part 1. Equations of State Derived from the van Der Waals Theory. Pure Components. Chem. Biochem. Eng. Q. 1999, 13 (3), 89-100.

(50) Kontogeorgis, G. M.; Coutsikos, P. Thirty Years with EoS $/ G^{\mathrm{E}}$ Models-What Have We Learned? Ind. Eng. Chem. Res. 2012, 51 (11), 4119-4142.

(51) Kontogeorgis, G. M.; Economou, I. G. Equations of State: From the Ideas of van Der Waals to Association Theories. J. Supercrit. Fluids 2010, 55 (2), 421-437.
(52) Renon, H.; Prausnitz, J. M. Local Compositions in Thermodynamic Excess Functions for Liquid Mixtures. AIChE J. 1968, 14 (1), 135-144.

(53) Abbott, M. M.; Prausnitz, J. M. Generalized van Der Waals Theory: A Classical Perspective. Fluid Phase Equilib. 1987, 37, $29-62$.

(54) Privat, R.; Privat, Y.; Jaubert, J.-N. Can Cubic Equations of State Be Recast in the Virial Form? Fluid Phase Equilib. 2009, 282 (1), $38-50$.

(55) Wong, D. S. H.; Sandler, S. I. A Theoretically Correct Mixing Rule for Cubic Equations of State. AIChE J. 1992, 38 (5), 671-680.

(56) Prausnitz, J. M.; Tavares, F. W. Thermodynamics of FluidPhase Equilibria for Standard Chemical Engineering Operations. AIChE J. 2004, 50 (4), 739-761.

(57) Vidal, J.; Bogdanić, G. Equations of State, Mixing Rules and Phase Equilibrium Calculations. Part 2. Mixing Rules and VaporLiquid Equilibrium Calculation. Chem. Biochem. Eng. Q. 1999, 13 (3), $101-125$.

(58) Michelsen, M. L.; Möllerup, J. M. Thermodynamic Models: Fundamentals \& Computational Aspects; Tie-Line Publications: Holte, 2004.

(59) Czerwienski, G. J.; Tomasula, P.; Tassios, D. Vapor - Liquid Equilibria with the VdW - 711 Equation of State. Fluid Phase Equilib. 1988, 42, 63-83.

(60) Jaubert, J.-N.; Privat, R.; Le Guennec, Y.; Coniglio, L. Note on the Properties Altered by Application of a Péneloux-Type Volume Translation to an Equation of State. Fluid Phase Equilib. 2016, 419, $88-95$.

(61) Privat, R.; Jaubert, J.-N.; Le Guennec, Y. Incorporation of a Volume Translation in an Equation of State for Fluid Mixtures: Which Combining Rule? Which Effect on Properties of Mixing? Fluid Phase Equilib. 2016, 427, 414-420.

(62) Sako, T.; Wu, A. H.; Prausnitz, J. M. A Cubic Equation of State for High-Pressure Phase Equilibria of Mixtures Containing Polymers and Volatile Fluids. J. Appl. Polym. Sci. 1989, 38 (10), 1839-1858.

(63) Kontogeorgis, G. M.; Harismiadis, V. I.; Fredenslund, A.; Tassios, D. P. Application of the van Der Waals Equation of State to Polymers. I. Correlation. Fluid Phase Equilib. 1994, 96, 65-92.

(64) Polishuk, I.; Wisniak, J.; Segura, H. Simultaneous Prediction of the Critical and Sub-Critical Phase Behavior in Mixtures Using Equation of State I. Carbon Dioxide-Alkanols. Chem. Eng. Sci. 2001, $56(23), 6485-6510$.

(65) Polishuk, I.; Wisniak, J.; Segura, H.; Yelash, L. V.; Kraska, T. Prediction of the Critical Locus in Binary Mixtures Using Equation of State. Fluid Phase Equilib. 2000, 172 (1), 1-26.

(66) Kontogeorgis, G. M.; Coutsikos, P.; Harismiadis, V. I.; Fredenslund, A.; Tassios, D. P. A Novel Method for Investigating the Repulsive and Attractive Parts of Cubic Equations of State and the Combining Rules Used with the VdW-1f Theory. Chem. Eng. Sci. 1998, 53 (3), 541-552.

(67) Voutsas, E.; Louli, V.; Boukouvalas, C.; Magoulas, K.; Tassios, D. Thermodynamic Property Calculations with the Universal Mixing Rule for EoS $/ \mathrm{G}^{\mathrm{E}}$ Models: Results with the Peng-Robinson EoS and a UNIFAC Model. Fluid Phase Equilib. 2006, 241 (1-2), 216-228.

(68) Schmid, B.; Gmehling, J. Present Status of the Group Contribution Equation of State VTPR and Typical Applications for Process Development. Fluid Phase Equilib. 2016, 425, 443-450.

(69) Harismiadis, V. I.; Koutras, N. K.; Tassios, D. P.; Panagiotopoulos, A. Z. How Good Is Conformal Solutions Theory for Phase Equilibrium Predictions? Fluid Phase Equilib. 1991, 65, 118.

(70) Hudson, G. H.; McCoubrey, J. C. Intermolecular Forces between Unlike Molecules. A More Complete Form of the Combining Rules. Trans. Faraday Soc. 1960, 56, 761-766.

(71) Coutinho, J. A. P.; Kontogeorgis, G. M.; Stenby, E. H. Binary Interaction Parameters for Nonpolar Systems with Cubic Equations of State: A Theoretical Approach. 1. $\mathrm{CO}_{2} /$ Hydrocarbons Using SRK Equation of State. Fluid Phase Equilib. 1994, 102 (1), 31-60. 
(72) Coutinho, J. A. P.; Vlamos, P. M.; Kontogeorgis, G. M. General Form of the Cross-Energy Parameter of Equations of State. Ind. Eng. Chem. Res. 2000, 39 (8), 3076-3082.

(73) Tihic, A. Group Contribution SPC-SAFT Equation of State. Ph.D. Thesis, Technical University of Denmark: Denmark, 2008.

(74) Haslam, A. J.; Galindo, A.; Jackson, G. Prediction of Binary Intermolecular Potential Parameters for Use in Modelling Fluid Mixtures. Fluid Phase Equilib. 2008, 266 (1-2), 105-128.

(75) Harismiadis, V. I.; Panagiotopoulos, A. Z.; Tassios, D. P. Phase Equilibria of Binary Lennard-Jones Mixtures with Cubic Equations of State. Fluid Phase Equilib. 1994, 94, 1-18.

(76) Coutsikos, P.; Magoulas, K.; Kontogeorgis, G. M. Prediction of Solid-Gas Equilibria with the Peng-Robinson Equation of State. J. Supercrit. Fluids 2003, 25 (3), 197-212.

(77) Prigogine, I. The Molecular Theory of Solutions; North-Holland; Amsterdam, 1957.

(78) Kontogeorgis, G. M.; Nikolopoulos, G. I.; Fredenslund, A.; Tassios, D. P. Improved Models for the Prediction of Activity Coefficients in Nearly Athermal Mixtures Part II. A TheoreticallyBased $G^{\mathrm{E}}$-Model Based on the van Der Waals Partition Function. Fluid Phase Equilib. 1997, 127 (1-2), 103-121.

(79) Polyzou, E. N.; Vlamos, P. M.; Dimakos, G. M.; Yakoumis, I. V.; Kontogeorgis, G. M. Assessment of Activity Coefficient Models for Predicting Solid-liquid Equilibria of Asymmetric Binary Alkane Systems. Ind. Eng. Chem. Res. 1999, 38 (1), 316-323.

(80) Elbro, H. S.; Fredenslund, A.; Rasmussen, P. A New Simple Equation for the Prediction of Solvent Activities in Polymer Solutions. Macromolecules 1990, 23 (21), 4707-4714.

(81) Oishi, T.; Prausnitz, J. M. Estimation of Solvent Activities in Polymer Solutions Using a Group-Contribution Method. Ind. Eng. Chem. Process Des. Dev. 1978, 17 (3), 333-339.

(82) Moebus, J. A.; Greenhalgh, B. R. Modeling Vapor Solubility in Semicrystalline Polyethylene. Macromol. React. Eng. 2018, 12 (4), 1700072 .

(83) Huron, M.-J.; Vidal, J. New Mixing Rules in Simple Equations of State for Representing Vapour-Liquid Equilibria of Strongly NonIdeal Mixtures. Fluid Phase Equilib. 1979, 3 (4), 255-271.

(84) Jaubert, J.-N.; Mutelet, F. VLE Predictions with the PengRobinson Equation of State and Temperature-Dependent $k_{i j}$ Calculated through a Group Contribution Method. Fluid Phase Equilib. 2004, 224 (2), 285-304.

(85) Xu, X.; Jaubert, J.-N.; Privat, R.; Arpentinier, P. Prediction of Thermodynamic Properties of Alkyne-Containing Mixtures with the E-PPR78 Model. Ind. Eng. Chem. Res. 2017, 56 (28), 8143-8157.

(86) Qian, J.-W.; Jaubert, J.-N.; Privat, R. Phase Equilibria in Hydrogen-Containing Binary Systems Modeled with the PengRobinson Equation of State and Temperature-Dependent Binary Interaction Parameters Calculated through a Group-Contribution Method. J. Supercrit. Fluids 2013, 75, 58-71.

(87) Jaubert, J.-N.; Privat, R.; Mutelet, F. Predicting the Phase Equilibria of Synthetic Petroleum Fluids with the PPR78 Approach. AIChE J. 2010, 56 (12), 3225-3235.

(88) Vitu, S.; Privat, R.; Jaubert, J.-N.; Mutelet, F. Predicting the Phase Equilibria of $\mathrm{CO}_{2}+$ Hydrocarbon Systems with the PPR78 Model (PR EoS and $\mathrm{k}_{\mathrm{ij}}$ Calculated through a Group Contribution Method). J. Supercrit. Fluids 2008, 45 (1), 1-26.

(89) Dahl, S.; Michelsen, M. L. High-Pressure Vapor-Liquid Equilibrium with a UNIFAC-Based Equation of State. AIChE J. 1990, 36 (12), 1829-1836.

(90) Holderbaum, T.; Gmehling, J. PSRK: A Group Contribution Equation of State Based on UNIFAC. Fluid Phase Equilib. 1991, 70 (2-3), 251-265.

(91) Boukouvalas, C.; Spiliotis, N.; Coutsikos, P.; Tzouvaras, N.; Tassios, D. Prediction of Vapor-Liquid Equilibrium with the LCVM Model: A Linear Combination of the Vidal and Michelsen Mixing Rules Coupled with the Original UNIFAC and the $t$-mPR Equation of State. Fluid Phase Equilib. 1994, 92, 75-106.
(92) Kontogeorgis, G. M.; Vlamos, P. M. An Interpretation of the Behavior of EoS $/ G^{\mathrm{E}}$ Models for Asymmetric Systems. Chem. Eng. Sci. 2000, 55 (13), 2351-2358.

(93) Soave, G. Application of Equations of State and the Theory of Group Solutions to Phase Equilibrium Prediction. Fluid Phase Equilib. 1993, 87 (1), 23-35.

(94) Soave, G. S.; Sama, S.; Oliveras, M. I. A New Method for the Prediction of VLE and Thermodynamic Properties. Preliminary Results with Alkane-Ether-Alkanol Systems. Fluid Phase Equilib. 1999, $156(1-2), 35-50$.

(95) Soave, G. S. Universal Method for Equations of State (UNIFEST): An Application of UNIFAC to Predict the Parameters of Cubic Equations of State. Fluid Phase Equilib. 2002, 193 (1), 7586.

(96) Soave, G. S.; Bertucco, A.; Vecchiato, L. Equation-of-State Group Contributions from Infinite-Dilution Activity Coefficients. Ind. Eng. Chem. Res. 1994, 33 (4), 975-980.

(97) Vidal, J. Cubic Equations of State for Reservoir Engineering and Chemical Process Design. Fluid Phase Equilib. 1989, 52, 15-30.

(98) Sacomani, P. A.; Brignole, E. A. Predictions of Activity Coefficients of Nearly Athermal Binary Mixtures Using Cubic Equations of State. Ind. Eng. Chem. Res. 2003, 42 (17), 4143-4145.

(99) Kontogeorgis, G. M.; Coutsikos, P. Comments on "Predictions of Activity Coefficients of Nearly Athermal Binary Mixtures Using Cubic Equations of State. Ind. Eng. Chem. Res. 2005, 44 (9), 33743375.

(100) Folas, G. K.; Berg, O. J.; Solbraa, E.; Fredheim, A. O.; Kontogeorgis, G. M.; Michelsen, M. L.; Stenby, E. H. High-Pressure Vapor-Liquid Equilibria of Systems Containing Ethylene Glycol, Water and Methane. Fluid Phase Equilib. 2007, 251 (1), 52-58.

(101) Zhong, C.; Masuoka, H. A New Mixing Rule for Cubic Equations of State and Its Application to Vapor-Liquid Equilibria of Polymer Solutions. Fluid Phase Equilib. 1996, 123 (1-2), 59-69.

(102) Zhong, C.; Masuoka, H. Modeling of Gas Solubilities in Polymers with Cubic Equation of State. Fluid Phase Equilib. 1998, 144 (1-2), 49-57.

(103) Vidal, J. Thermodynamics: Applications in Chemical Engineering and the Petroleum Industry; Institut français du pétrole publications; ed.s Technip: Paris, 2003.

(104) Tsonopoulos, C.; Heidman, J. L. High-Pressure Vapor-Liquid Equilibria with Cubic Equations of State. Fluid Phase Equilib. 1986, 29, 391-414.

(105) Hendriks, E. M. Applied Thermodynamics in Industry, a Pragmatic Approach. Fluid Phase Equilib. 2011, 311, 83-92.

(106) Zeck, S. Thermodynamics in Process Development in the Chemical Industry - Importance, Benefits, Current State and Future Development. Fluid Phase Equilib. 1991, 70 (2-3), 125-140.

(107) Gupta, S.; Olson, J. D. Industrial Needs in Physical Properties. Ind. Eng. Chem. Res. 2003, 42 (25), 6359-6374.

(108) Le Guennec, Y.; Lasala, S.; Privat, R.; Jaubert, J.-N. A Consistency Test for $\alpha$-Functions of Cubic Equations of State. Fluid Phase Equilib. 2016, 427, 513-538.

(109) Le Guennec, Y.; Privat, R.; Jaubert, J.-N. Development of the Translated-Consistent $t c$-PR and $t c$-RK Cubic Equations of State for a Safe and Accurate Prediction of Volumetric, Energetic and Saturation Properties of Pure Compounds in the Sub- and Super-Critical Domains. Fluid Phase Equilib. 2016, 429, 301-312.

(110) Le Guennec, Y.; Privat, R.; Lasala, S.; Jaubert, J.-N. On the Imperative Need to Use a Consistent $\alpha$-Function for the Prediction of Pure-Compound Supercritical Properties with a Cubic Equation of State. Fluid Phase Equilib. 2017, 445, 45-53.

(111) Pina-Martinez, A.; Le Guennec, Y.; Privat, R.; Jaubert, J.-N.; Mathias, P. M. Analysis of the Combinations of Property Data That Are Suitable for a Safe Estimation of Consistent Twu $\alpha$-Function Parameters: Updated Parameter Values for the Translated-Consistent $T_{c}$-PR and Tc-RK Cubic Equations of State. J. Chem. Eng. Data 2018, 63 (10), 3980-3988.

(112) Palma, A. M.; Oliveira, M. B.; Queimada, A. J.; Coutinho, J. A. P. Re-Evaluating the CPA EoS for Improving Critical Points and 
Derivative Properties Description. Fluid Phase Equilib. 2017, 436, 8597.

(113) Palma, A. M.; Queimada, A. J.; Coutinho, J. A. P. Modeling of the Mixture Critical Locus with a Modified Cubic Plus Association (CPA) EoS: Aromatics, Ketones, Ethers, Diethyl Carbonate, and THF. Ind. Eng. Chem. Res. 2018, 57 (46), 15857-15868.

(114) Palma, A. M.; Queimada, A. J.; Coutinho, J. A. P. Modeling of the Mixture Critical Locus with a Modified Cubic Plus Association

Equation of State: Water, Alkanols, Amines, and Alkanes. Ind. Eng.

Chem. Res. 2018, 57 (31), 10649-10662. 